\title{
1 Neural encoding of phrases and sentences in spoken 2 language comprehension
}

3

4

5

7

8

Fan Bai ${ }^{1,2}$, Antje S. Meyer ${ }^{1,2}$, and Andrea E. Martin ${ }^{1,2, *}$

1, Max Planck Institute for Psycholinguistics, Nijmegen, The Netherlands;

2, Donders Institute for Brain, Cognition, and Behaviour, Radboud University, Nijmegen, The Netherlands

Corresponding author:

Andrea E. Martin; andrea.martin@mpi.nl;

14

(16

(1)

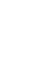

2

4

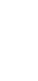

7

0

(1)

(3)

4

5




\section{Abstract}

Speech stands out in the natural world as a biological signal that communicates formallyspecifiable complex meanings. However, the acoustic and physical dynamics of speech do not injectively mark the linguistic structure and meaning that we perceive. Linguistic structure must therefore be inferred through the human brain's endogenous mechanisms, which remain poorly understood. Using electroencephalography, we investigated the neural response to synthesized spoken phrases and sentences that were closely physically-matched but differed in syntactic structure, under either linguistic or non-linguistic task conditions. Differences in syntactic structure were well-captured in theta band ( 2 to $7 \mathrm{~Hz}$ ) phase coherence, phase connectivity degree at low frequencies $(<\sim 2 \mathrm{~Hz})$, and in both intensity and degree of power connectivity of induced neural response in the alpha band ( 7.5 to $13.5 \mathrm{~Hz}$ ). Theta-gamma phase-amplitude coupling was found when participants listened to speech, but it did not discriminate between syntactic structures. Spectral-temporal response function modelling

52 suggested different encoding states in both temporal and spectral dimensions as a function of

53 the amount and type of linguistic structure perceived, over and above the acoustically-driven neural response. Our findings provide a comprehensive description of how the brain separates

55 linguistic structures in the dynamics of neural responses, and imply that phase synchronization and strength of connectivity can be used as readouts for constituent structure, providing a novel basis for future neurophysiological research on linguistic structure in the brain. 


\section{Introduction}

60 Speech contains an abundance of acoustic features in both the time and frequency domains

61 (Shannon, Zeng, Kamath, Wygonski, \& Ekelid, 1995; Smith, Delgutte, \& Oxenham, 2002; Zeng et

62 al., 2005). While these features are crucial for speech comprehension, they do not themselves

63 signpost the linguistic units and structures that give rise to meaning. Spoken language

64 comprehension therefore relies on listeners to go beyond the information given and infer the

65 presence of linguistic structure based on their knowledge of language. As such, many theories

66

67

68 posit that linguistic structures - ranging from syllables to 'words' to syntactic structures - are constructed via an endogenous inference process (Bever \& Poeppel, 2010; Brown, Tanenhaus, \& Dilley, 2021; Friederici, 1995; Hagoort, 2013; Halle \& Stevens, 1962; Marslen-Wilson \& Tyler, 1980; Marslen-Wilson, 1987; Marslen-Wilson \& Welsh, 1978; Martin, 2016, 2020; Martin \& Doumas, 2017, 2019, 2020; Meyer, Sun, \& Martin, 2020; Phillips, 2003; Poeppel \& Monahan, 2011). On this view, also known as 'analysis-by-synthesis' (Halle, Stevens, Wathen-Dunn, \& Woods, 1959), speech triggers internal generation of memory representations (synthesis), which are compared to the sensory input (analysis). This results in linguistic structures that come about through the synthesis of internal brain states (viz., linguistic knowledge) with sensory representations via perceptual inference (Marslen-Wilson \& Welsh, 1978; Martin, 2016, 2020). Recent studies have begun to investigate the neural activity that corresponds to the emergence of linguistic structure (e.g., Ding, Melloni, Zhang, Tian, \& Poeppel, 2016; Kaufeld et al., 2020; Keitel, Gross, \& Kayser, 2018; Meyer \& Gumbert, 2018), in particular in terms of temporal and spatial dynamics of brain rhythms. However, despite these efforts, details of how the brain encodes or distinguishes syntactic structures remain unknown. In this study, we 
81 investigated the neural responses to minimally different linguistic structures, specifically

82 phrases like 'the red vase' compared to sentences like 'the vase is red'. We investigated which

83

84

85

dimensions of neural activity distinguish the linguistic structure of these phrases and sentences, by minimizing their differences in acoustic-energetic/temporal-spectral profiles and semantic components .

\section{Low frequency neural oscillations and linguistic structure}

A growing neuroscience literature suggests that low frequency neural oscillations $(<8 \mathrm{~Hz})$ are involved in processing linguistic structures (Brennan \& Martin, 2020; Brodbeck, Hong, \& Simon, 2018; Ding, Melloni, et al., 2017; Ding et al., 2016; Gui et al., 2020; Gwilliams \& King, 2020; Jin, Lu, \& Ding, 2020; Jin, Zou, Zhou, \& Ding, 2018; Kaufeld et al., 2020; Keitel et al., 2018; Martin \& Doumas, 2017, 2019; Meyer, 2018; Meyer \& Gumbert, 2018; Obleser \& Kayser, 2019; Zhou, Melloni, Poeppel, \& Ding, 2016). In a highly influential study by Ding and colleagues (2016), low frequency power 'tagged' the occurrence of phrases and sentences in controlled speech stimuli.

That is, power increases were observed that coincided with occurences of phrases or sentences at a fixed rate of 2 and $1 \mathrm{~Hz}$, respectively. Additionally, the grouping of words into phrases of different lengths modulated the location of the frequency tag accordingly, indicating that power at certain frequencies could track linguistic structures. Subsequent research has further confirmed the sensitivity of oscillatory power and phase in the delta band ( $2-4 \mathrm{~Hz})$ to higher level linguistic structures like phrases (Brennan \& Martin, 2020; Kaufeld et al., 2020; Meyer \& Gumbert, 2018; Meyer, Henry, Gaston, Schmuck, \& Friederici, 2017; Peña \& Melloni, 2012). 
Inspired by these empirical studies, Martin and Doumas (2017) recently provided a

112 The current study tested this prediction using Dutch phrases (e.g., de rode vas, 'the red vase,'

\footnotetext{
${ }^{1}$ Their model exploited asynchrony of unit firing to form structures from words, in which the temporal proximity of unit firing was used to encode linguistic structures and relations. More specifically, nodes on higher layer (i.e., localist units representing phrasal units) code for the composition of sub-layer units (i.e., words) in this network structure. The higher-level node fires when any of its sublayer nodes fire in time, forming a 'phase set' between words and their phrase. In this implementation, linguistic structures were represented neural dynamics across a layered network, where the asynchrony of unit firing (firing staggered in time) not only allowed the network to combine lower-level representations together for the processing on a higherlevel, but also served to keep the lower-level representations independent as well (Doumas et al., 2008; Martin \& Doumas, 2017, 2019).
} 
117 in syntactic structure (viz., the number and type of constituents). Fig 1 shows the syntactic

118 structures in the sample phrase-sentence pair, de rode vaas (the red vase) and de vaas is rood

119 (the vase is red) in a minimalistic syntactic denotation.

Fig 1. Syntactic structure comparison between phrase and sentence. (a) Tree representation of the

\section{Low frequency oscillations and speech intelligibility}

Low frequency neural activity, in particular phase coherence in the $\sim 2-7 \mathrm{~Hz}$ theta band,

is highly correlated with spoken language comprehension (e.g., Doelling, Arnal, Ghitza, \& 
148 (Doelling et al., 2014; Howard \& Poeppel, 2010; Kösem \& van Wassenhove, 2017; Luo \&

Poeppel, 2007; Peelle et al., 2013). However, the format in which the brain represents higher-

151 substantial implications for theories of speech comprehension.

\section{The current study}

We investigated whether low frequency neural oscillations reflect differences in

syntactic structure. In order to increase the likelihood that any observed patterns are due to 
160 De vaas is rood (The vase is red), which combine a subject with a verb into a proposition.

161 Phrases and sentences were matched in the number of syllables (4 syllables), the semantic

162 components (same color and object), the duration in time (1-second, sampling rate $44.1 \mathrm{k} \mathrm{Hz}$ ),

163 and the overall energy (Root Mean Squared value equals $-16 \mathrm{~dB}$ ).

We formulated a general hypothesis that low frequency neural oscillations would be

sensitive to the difference in syntactic structure of the phrases and sentences. However, we did

not limit our analysis to low frequency power and phase, as in previous research (Brennan \&

167 Martin, 2020; Ding et al., 2016; Kaufeld et al., 2020; Keitel et al., 2018). We hypothesized that

the neural response difference between phrases and sentences may manifest itself in a number

of dimensions, dimensions that are outside of the view of typical analyses of low frequency power and phase.

We therefore employed additional methods to decompose the neural response to

172 phrases and sentences, to address the following five questions:

Question 1: Do phrases and sentences have different effects on brain dynamics as

174 reflected at the functional neural network level (viz., functional connectivity). Neuroscience is

175 rapidly growing interest in investigating functional connectivity to study whole brain dynamics

176 in sensor space (Cabral, Kringelbach, \& Deco, 2014; Cohen, 2014, 2015; Hutchison et al., 2013;

177 Sporns, 2010), which can reveal temporal synchronization (viz., phase coherence) between

178 brain regions. Neurophysiological techniques such as EEG and MEG have a high temporal

179 resolution and are suitable for calculating synchronization across frequency bands in functional

180 brain networks (Stam, Nolte, \& Daffertshofer, 2007). Describing the temporal synchronization 
of the neural activity over the whole brain is the first step in decomposing neural responses to

high-level variables like syntactic structure. We therefore investigated whether phrases and sentences have different effects on inter-site phase coherence (ISPC), which is considered to reflect the temporal synchronization of neural activity (Cohen, 2014; Lachaux et al., 2000; Mormann, Lehnertz, David, \& Elger, 2000).

Question 2: Do phrases and sentences differ in the intensity with which they engage connected brain regions? Power connectivity (Cohen, 2011, 2014, 2015) can be used to describe a functional neural network by the energy that is engaged in a cognitive task. Power connectivity is a measure of how different underlying brain regions are connected via the intensity of induced neural responses in the time-frequency space. Differences in power connectivity would imply that phrases and sentences differently impact the distribution and intensity of neural networks involved in speech comprehension.

Question 3: Do phrases and sentences have different effects on the coupling between lower and higher frequency activity? This question is related to the theoretical model by Giraud and Poeppel (2012) of a generalized neural mechanism for speech perception below the level of the word. The model, which is focused on syllable-level processing, suggests that presentation of the speech stimulus first entrains an inherent neural response at low frequencies (less than 8 $\mathrm{Hz}$ ) in order to track to the speech envelope, from which the neural representation of syllables

199 is then constructed. Then, the low frequency neural response evokes a neural response at a 200 higher-frequency (25 to $35 \mathrm{~Hz}$ ), which reflect the brain's analysis of phonemic level information.

201 The model proposes coupling between the low and the high frequency neural responses (theta and gamma, respectively) as the fundamental neural mechanism for speech perception up to 
the syllable. We therefore investigated whether theta-gamma frequency coupling may also differentiate higher-level linguistic structure, namely phrases and sentences.

Question 4: Do phrases and sentences also differentially impact neural activity at higher frequencies such as the alpha band? The functional role of alpha band oscillations in perception and memory processing is currently widely debated in systems neuroscience. However, whereas the role for low frequency neural activity in language processing is beyond doubt, whether alpa band activity has an important contribution is not yet agreed upon. Alpha band activity correlates with verbal working memory (Haegens, Osipova, Oostenveld, \& Jensen, 2010; Obleser, Wöstmann, Hellbernd, Wilsch, \& Maess, 2012; Ten Oever, De Weerd, \& Sack, 2020; Wilsch \& Obleser, 2016) and auditory attention (Strauß, Wöstmann, \& Obleser, 2014; Wöstmann, Herrmann, Maess, \& Obleser, 2016; Wöstmann, Herrmann, Wilsch, \& Obleser, 2015; Wöstmann, Lim, \& Obleser, 2017). Some oscillator models of speech perception consider the induced neural response at alpha as a "top-down" gating control signal (Ghitza, Giraud, \& Poeppel, 2013; Giraud \& Poeppel, 2012), reflecting domain-general aspects of perceptual processing that are not specific to language processing. Other researchers argue that alpha activity reflects speech intelligibility (Becker, Pefkou, Michel, \& Hervais-Adelman, 2013; Dimitrijevic, Smith, Kadis, \& Moore, 2017; Obleser \& Weisz, 2012), which opens up a potential role for alpha band oscillations in syntactic or semantic processing. We therefore investigated whether phrases and sentences elicited differences in alpha band activity.

Question 5: Can we obtain evidence for the differential encoding of phrases and sentences after modelling out physical differences? Neural responses to phrases and sentences comprise a mixture of processes associated with linguistic structure building and with 
processing acoustic differences. To deal with this issue, one can model which aspects of the neural response encode acoustic differences, and then detect differences between phrases and sentences in the remainder of the neural activity, from which acoustic differences have been regressed out. Previous research using this approach, the spectral temporal response function (STRF), shows that low frequency neural responses robustly represent the acoustic features in the speech (Ding \& Simon, 2012a, 2012b, 2013) and that phonemic level processing is reflected in the low frequency entrainment to speech (Di Liberto, O'Sullivan, \& Lalor, 2015; Donhauser \& Baillet, 2020; Weissbart, Kandylaki, \& Reichenbach, 2020). We therefore used the STRF to investigates which dimensions of the neural responses reflect differences between phrases and sentences.

In sum, we investigated different dimensions of the electroencephalography (EEG) response to spoken phrases and sentences. Observing differences between phrases and sentences would serve as a trailmarker on the path towards a theory of the neural computations underlying of syntactic structure formation.

\section{Results}

\section{Low frequency phase coherence distinguishes phrases and sentences}

To answer our first question, whether the low frequency neural oscillations distinguish phrases and sentences, we calculated the phase coherence (for details see Methods). We then performed non-parametric cluster-based permutation tests (1000 permutations) on a $1200-\mathrm{ms}$ time window starting at the audio onset and over the frequencies from $1 \mathrm{~Hz}$ to $8 \mathrm{~Hz}$. The comparison indicated that phase coherence was significantly higher for sentences than phrases 
$246(p<1 e-4$, two-tailed). In the selected latency and frequency range, the effect was most

247 pronounced at central electrodes.

248 Fig 2a shows the temporal evolution, in steps of $50 \mathrm{~ms}$, of this condition effect which is computed as the phase coherence of the phrase condition minus the phrase coherence of the sentence condition. Fig $\mathbf{2} \mathbf{b}$ shows the time-frequency plot using all the sensors in this cluster, in which the upper and lower panel are the plot for the phrase condition and the sentence condition, respectively. phrases and sentences, consistent with the hypothesis that low frequency phase coherence represents cortical computations over speech stimuli (Brennan \& Martin, 2020; Doelling et al., comprehension at the level of syntactic processing.

Fig 2. Results of phase coherence. Statistical analysis for comparing the phase coherence (ITPC) difference between phrases and sentences was conducted by using the non-parametric cluster based permutation test (1000 times) on a 1200 -ms time window starting at the audio onset and over the frequencies from $2 \mathrm{~Hz}$ to $8 \mathrm{~Hz}$. The results indicated that the phase coherence was higher for the sentences than the phrases ( $p<1 e-4 * * *$, two-tailed). (a) The temporal evolution of the cluster that corresponds to the condition difference between phrases and sentences. The activity was drew by using the ITPC of the 


\section{Low frequency (< $2 \mathrm{~Hz}$ ) phase connectivity degree over the sensor} space separates phrases and sentences

We initially calculated phase connectivity over the sensor space by ISPC at each time-

273 frequency bin (for details see Methods section). We then used a statistical threshold method to

274 transform each connectivity representation to a super-threshold count at each bin. After

275 baseline correction, we conducted a 1000 time cluster-based permutation test on a 3500-ms

276 time window starting at the audio onset and over the frequencies from $1 \mathrm{~Hz}$ to $8 \mathrm{~Hz}$ to compare

277 the degree of the phase connectivity between phrases and sentences (for details see Methods).

278 Phrases and sentences showed a significant difference in connectivity $(p<0.01$, two-tailed). The

279 effect corresponded to a cluster extended from $\sim 1800 \mathrm{~ms}$ to $\sim 2600 \mathrm{~ms}$ after the speech

280 stimulus onset, and was mainly located at a very low frequency range $(<\sim 2 \mathrm{~Hz})$. In the selected

281 latency and frequency range, the effect was most pronounced at the right posterior region.

282 Fig 3a shows the temporal evolution of the condition effect, which is represented by the connectivity degree of the phrase condition minus the connectivity degree of sentence

284 condition (in steps of $100 \mathrm{~ms}$ ). Fig $3 \mathbf{b}$ shows the time-frequency plot of the phase connectivity

285 degree, which is averaged across all sensors in this cluster. The left and right panel are the time-

286 frequency plot for the phrase condition and the sentence condition, respectively. 
Since the statistical analysis indicated a phase connectivity degree difference between phrases and sentences, we assessed how this effect was distributed in the sensor space. To do so, we extracted all binarized connectivity matrices that corresponded to the time and

290 frequency range of the cluster and averaged all the matrices in this range for both conditions

291 (details see Methods). Fig 3c shows the averaged matrix representation of the sentence

292 condition minus the averaged matrix representation of the phrase condition. This result

293 suggests that the connectivity difference was mainly localized at the frontal-central area. After

294 extracting the matrix representation, we used all sensors of this cluster as seeds to plot

297 panel represent the phrase and the sentence condition, respectively. The figure shows how the

298 phase connectivity (synchronization) is distributed on the scalp in each condition. From this

299 figure we can see that the overall degree of the phase connectivity was stronger for the

300 sentence condition than the phrase condition. that the effect was most prominent at the right posterior region. 
phase connectivity degree was higher for the sentences than the phrases $\left(p<0.01^{* *}\right.$, two-tailed). (a) The temporal evolution of

\section{Phase Amplitude Coupling (PAC) as a generalized neural mechanism} for speech perception

To assess whether PAC distinguished phrases and sentences, we calculated the PAC 
332 there was a strong low frequency phase $(4 \mathrm{~Hz}$ to $10 \mathrm{~Hz})$ response entrained to high frequency

333 amplitude $(15 \mathrm{~Hz}$ to $40 \mathrm{~Hz})$. The results indicate that the PAC was introduced when participants

334 listened to the speech stimuli.

Fig $\mathbf{4 b}$ shows how the sensors were selected. The larger the red circle indicates the more often the sensor was selected across participants.

Fig 4c shows the topographical representation of the PAC-Z. The activity in these figures was the averaged PAC-Z values over the frequency range for phase (4 to $10 \mathrm{~Hz}$ ) and the frequency range for amplitude $(15$ to $40 \mathrm{~Hz}$ ). The results indicate that when the participants

340 listened to the speech stimuli, PAC was introduced symmetrically at both hemispheres over the 341 central area. This could be evidence for the existence of PAC when speech stimuli are being

342 processed. However, both the parametric and the non-parametric statistical analysis failed to show a significant difference of the PAC-Z between phrases and sentences, which means we do

344 not have evidence to show that the PAC was related to syntactic information processing.

345 Therefore, our results suggest the PAC could be a generalized neural mechanism for speech perception, rather than a mechanism specifically recruited during the processing of higher-level

347 linguistic structures. 
showed the biggest PAC-Z over the region which defined by the phase frequency from 4 to $10 \mathrm{~Hz}$ and the amplitude frequency from 15 to $40 \mathrm{~Hz}$. A z-score transformation with Bonfferroni correction was conducted to test the significance, which lead to the threshold to be 3.73 corresponding to $p=0.05$. (b) The figure shows how sensors were selected at each hemisphere. The bigger the red circle indicates the more times this sensor was selected. (c) The topographical distribution of the PAC-Z. The figure indicates that the PAC was largely localized at the bilateral central areas.

\section{Alpha band inhibition reflects syntactic structure}

To query whether neural oscillations at alpha band reflect the processesing of syntactic structure, we calculated the induced power. The grand average (over all participants and all conditions) of the induced power showed a strong inhibition at the alpha band ( 7.5 to 13.5 $\mathrm{Hz}$ ). Therefore, we checked whether this alpha band inhibition could separate the two types of linguistic structures. Statistical analysis was conducted using the nonparametric cluster-based permutation test (1000 times) over the frequencies of alpha band in the 1000 ms time window from the audio onset for details see Methods). The results indicated that the alpha band inhibition was stronger for the phrase condition than the sentence condition ( $p<0.01$, twotailed). In the selected time and frequency range, this effect corresponded to a cluster that lasted from $\sim 350$ ms to $\sim 1000$ ms after the audio onset and was largely localized at the left hemisphere, though the right frontal-central sensors were also involved during the temporal evolution of this cluster. Fig 5a shows the temporal evolution of this cluster in steps of $50 \mathrm{~ms}$ using the induced power of the phrase condition minus the induced power of the sentence condition. Fig $\mathbf{5 b}$ shows the time-frequency plot of the induced power using the average of all the sensors in this cluster. The upper and lower panel shows the phrase condition and the sentences condition, respectively. From these figures, we can see that the alpha band inhibition 
was stronger for the phrase condition than the sentence condition. These results show that the processing of phrases and sentences is reflected in the intensity of the induced neural response

377 in the alpha band.

Fig 5. Results of induced neural response. Statistical analysis for comparing the induced power difference between

phrases and sentences was conducted by using the non-parametric cluster based permutation test (1000 times) on a 1000-ms coherence was higher for sentences than phrases $(p<0.01 * *$, two-tailed). (a) The temporal evolution of the cluster that corresponds to the condition difference between phrases and sentences. The activity was drew by using the induced power of the phrase condition minus the induced power of sentence condition. The topographies were plotted in steps of $50 \mathrm{~ms}$. (b) the phrase condition and the sentence condition, respectively. level separation of phrases and sentences 
up, which resulted in four conditions for each participant (left-phrase, left-sentence, rightphrase and right-sentence).

Fig 6a shows the power connectivity degree which was averaged over all participants for each condition. To check whether power connectivity degree separated the phrases and the sentences, a Stimulus-Type*Hemisphere 2-way repeated measure ANOVA was conducted. The comparison revealed a main effect of Stimulus-Type $(F(1,14)=5.28, p=0.033)$. Planned post hoc comparison using paired sample t- tests on the main effect of the Stimulus-Type showed that the power connectivity inhibition was stronger for the phrases than the sentences $(t(29)=$ sensor space using the average of the binarized sentence connectivity matrix minus the average connectivity was stronger for phrases than for sentences. In other words, the overall level of

415 the power connectivity was higher for sentences than for phrases. Fig $6 \mathrm{e}$ is the topographical

416 representation of the power connectivity, which was plotted using the binarized power

417 connectivity of the selected sensors. The upper and lower panel are the phrase condition and

418 the sentence condition, respectively. From this figure, we can see that the difference was 
419 largely localized at the bilateral central area, and more strongly present at the left than the right hemisphere. These results reflect that the neural network which was organized by the intensity of the induced power at alpha band was different for the two syntactic structures.

Fig 6. Results of power connectivity. (a) Power connectivity degree for all conditions. The left and right figure of the upper and lower panel shows the power connectivity degree for the phrase condition and the sentence condition at the left and right hemisphere, respectively. Each plot was clustered by the sensors at each hemisphere that showed the biggest inhibition level of the grand averaged power connectivity. (b) The results of two-way repeated measure ANOVA of power connectivity on the factors of stimulus-type (phrase or sentence) and hemisphere (left or right) indicated a significant main effect of stimulus-type, post hoc comparison on the main effect indicated that the overall inhibition level of the power connectivity was stronger for the phrases than the sentences $\left(\mathrm{t}(29)=2.82, \mathrm{p}=0.0085^{* *}\right.$, two-sided). (c) How sensors were binarized connectivity matrix of the sentence condition minus the phrase condition. The figure shows that the connectivity degree over the sensor space for the sentence condition was higher than the phrase condition. (e) Topographical plot of the binarized connectivity that was clustered by the sensors showing biggest inhibition level of the power connectivity. The upper and lower panel shows the phrase and sentence condition, respectively.

\section{Different encoding states for phrases versus sentences in both} temporal and spectral dimensions

Previous research has shown that the low frequency neural response reliably reflect the 
443

444

445

446

447

448

449

450

451

452

453

condition at all frequency bands, which are Delta (<4 Hz), Theta (4 to $7 \mathrm{~Hz}$ ), Alpha (8 to $13 \mathrm{~Hz}$ ),

Beta (14 to $30 \mathrm{~Hz}$ ) and Low-Gamma (31 to $50 \mathrm{~Hz}$ ), respectively. Then we compared the real performance of the STRFs to the random performance of them (for details see Methods). Fig 7a shows the results of this comparison. The blue and red dots represent the real performance of the STRFs, the error bar presents 1 s.e.m on each side. The small gray dots represents the random performance (1000 times in each frequency band per condition). The upper boarder, which is delineated by these gray dots represents the 97.5 percentiles of the random performance. The performance of the STRFs was above chance level only at the low frequency (Delta and Theta) band, which was consistent with previous research (Ding \& Simon, 2012a, 2012b). Our results verified that the low frequency STRF reliably reflected the relationship between the acoustic features of speech and the neural response at low frequencies.

Since only low frequency neural responses robustly reflected the encoding of the speech stimuli, we fitted the STRF for both conditions using the neural response that was low passed at $9 \mathrm{~Hz}$. Leave-one-out cross validation was used to maximize the performance of the STRFs (for details see Methods). Fig 7b shows the performance of the STRF for each condition. The transparent dots, blue for phrases and red sentences, represent the model's performance on each testing trial. The solid dots represent the model's performance that was averaged over all trials, the error bars represent 1 s.e.m on each side of the mean. A paired sample t-test was used to compare the performance between the phrase condition and the sentence condition. No evidence was shown to indicate a performance's difference between these two conditions ( $t$ $(74)=1.25, p=0.21)$. The results indicate that the STRF were fitted equally well for phrases and sentences. Thus any difference in temporal-spectral features between the STRF of phrases and 
sentences cannot be driven by the model's performance. Fig 7c shows the comparison between the real neural response and the model predicted response at the sample sensor $\mathrm{Cz}$. The upper and lower panel shows the performance of the STRF to phrases $(r=0.47, N=1024, p<1 e-5)$ and sentences $(r=0.41, N=1024, p<1 e-5)$, respectively.

The grand average of the STRFs was negative from 0 to $400 \mathrm{~ms}$ in the time domain and from 100 to $1000 \mathrm{~Hz}$ in the frequency domain, and the sensor clustering of the STRF was conducted based on the averaged activation these domain periods. More concretely, we selected eight sensors at each hemisphere for each participant, which showed the strongest averaged magnitude (negative) in this region. Fig 7d shows the clustered STRFs that were averaged across all participants. Fig 7e shows how the sensors were selected across the participants, in which the bigger the red circle indicates the more times a given sensor was selected.

To compare the differences of the kernel (STRF) in both the temporal and spectral dimensions, the TRF and the SRF were extracted separately for each condition (for details see Methods).

Fig $7 f$ shows the TRFs that were averaged across all participants. The grand average of all TRFs showed two peaks at $\sim 100 \mathrm{~ms}$ and $\sim 300 \mathrm{~ms}$. We therefore defined the first temporal window from 50 to $150 \mathrm{~ms}$ (center at $100 \mathrm{~ms}$ ) and the second temporal window from 250 to 350 (center at $300 \mathrm{~ms}$ ) for the search for the magnitude and the latency of these two peaks. The latency of each peak was defined as the time when it appeared. The magnitude of each peak was defined as the average magnitude over a $5 \mathrm{~ms}$ window on both sides around it. After extracting the 
magnitude and the latency of these two peaks, a Stimulus-type*Peak-type*Hemisphere 3-way repeated measure ANOVA was conducted on both the magnitude and the latency.

For the magnitude of the TRF (Fig 7g), the statistical comparison showed a significant main effect of Stimulus-type $(F(1,14)=13.58, P=0.002)$ and a significant 3-way, Stimulustype*Peak-type*Hemisphere, interaction $(F(1,14)=15.25, P=0.001)$.

The post hoc comparison on the main effect of Stimulus-Type using paired-sample t-tests showed that the magnitude for phrases was significantly stronger than the magnitude for sentences $(t(59)=4.55, P<2 e-5)$. The results suggest that the instantaneous neural activity in response to phrases had a stronger phase-locked dependency on the acoustic features than in response to sentences.

To investigate the 3-way, Stimulus-type*Peak-type*Hemisphere, interaction, two 2-way repeated measure ANOVA with the Bonferroni correction were conducted on the factors of Hemisphere and Stimulus-type at each level of the Peak-type. The results indicated a main effect of Stimulus-Type at the first peak $(F(1,14)=8.19, p=0.012)$ and a 2-way Hemisphere*Stimulus-Type interaction at the second peak $(F(1,14)=6.42, p=0.023)$.

At the first peak, we conducted a post hoc comparison on the main effect of stimulustype using a paired sample t tests, which showed that the magnitude of the phrase condition was higher than the magnitude of the sentence condition $(t(29)=3.49, p=0.001)$. The results indicate that the neural activity was more strongly driven by the acoustic features that were presented $\sim 100$ ms ago when phrases than when sentences were presented. 
For the 2-way, Hemisphere*Stimulus-Type, interaction at the second peak, the paired-

507

508

509

510

511

512

513

514

515

516

sample t-tests with Bonferroni correction was conducted to compare the difference of the magnitude between phrases and sentences at each hemisphere. The results indicate that the magnitude at the second peak was stronger for phrases than sentences in the right hemisphere $(t(14)=3.21, p=0.006)$, but not the left hemisphere $(t(14)=0.86, p=0.40)$. The findings suggest that, at the right hemisphere, the instantaneous neural activity of the phrases was more strongly driven by the acoustic features that were present $~ 300 \mathrm{~ms}$ than it was under sentences.

For the latency of the TRF (Fig 7h), the comparison showed a main effect of the Peak-type (F (1, $14)=1 e+3, p<1 e-14)$ and a 3-way, Stimulus-type*Peak-type*Hemisphere, interaction $(F(1,14)$ $=8.04, p=0.013)$ The post hoc comparison for the main effect of the Peak-type with paired sample tests showed, as expected, that the latency of the first peak was significantly shorter than the second one $(t(59)=38.89, p<2 e-16)$. The result is clear since regardless of search method for the analysis time windows, the latency of the first one will always shorter than the second one. To investigate the 3-way, Stimulus-type*Peak-type*Hemisphere, interaction, two 2-way repeated measures ANOVA with the Bonferroni correction were conducted on the factors of Hemisphere and stimulus-type for each level of the Peak-type. The comparison suggested a 2way Hemisphere*Stimulus-Type interaction at the first peak $(F(1,14)=12.83, p=0.002)$. The post hoc comparison on this 2-way interaction using paired sample t-tests with the Bonferroni correction indicated that the latency at the first peak was significantly longer for sentences than 
527 for phrases at the right hemisphere $(t(14)=3.55, p=0.003)$, but not the left hemisphere $(t(14)$

$528=0.58, p=0.56)$. The results suggest that, within the first temporal window ( 50 to $150 \mathrm{~ms}$ ),

529 only at the right hemisphere, the neural response of the sentences was dominantly driven by

530 the acoustic features earlier in time than the acoustic features that drove the neural response

531 of the phrases.

Fig $7 \mathbf{i}$ shows the SRFs that were averaged across all participants. The grand average of the STRFs suggested that the activation of the kernel was most prominent in the frequency

534 range from $0.1 \mathrm{kHz}$ to $0.8 \mathrm{kHz}$. To compare the differences of the encoding of the acoustic

535 features in the spectral dimension, we separated the SRF into three frequency bands, which

536 were lower than $0.1 \mathrm{kHz}, 0.1$ to $0.8 \mathrm{kHz}$ and higher than $0.8 \mathrm{kHz}$. We then averaged the

537 response in each extracted frequency band for each condition. The statistical comparison was

538 conducted using 3-way repeated measure ANOVA on the factors of Hemisphere, stimulus-type

539 and Band-type. The results (Fig 7j) indicated a main effect of Band-type $(F(2,28)=119.67, p<$

$5402 e-14)$ and a 2-way, Band-type*stimulus-type, interaction $(F(2,28)=27.61, p<3 e-7)$.

542 with Bonferroni correction showed that the magnitude of the middle frequency band was

543 stronger than that of the low frequency band $(t(59)=17.9, p<4 e-25)$ and high frequency band

$544(t(59)=18.7, p<5 e-26)$. The results indicate that the acoustic features from different frequency

545 bands contributed differently to the evoked neural response. In other words, for both

546 conditions, the neural response was dominantly driven by the encoding of the acoustic features

547 from $0.1 \mathrm{kHz}$ to $0.8 \mathrm{kHz}$, which was considered as the spectral-temporal features at the range of

548 the first formant (Catford, 1988; Jeans, 1968; Titze et al., 2015; Titze \& Martin, 1998). 
Fig 7. Results of STRF. (a) The comparison between the real performance and the random performance of the STRF in each was better than the random performance. The blue and red dots represent the real performance of the STRFs for the phrase condition and the sentence condition, respectively. The error bar represents two S.E.M across the mean. The gray dots represents the random performance. (b) The performance of the low frequency $(<8 \mathrm{~Hz})$ STRF which was averaged across all participants. The solid blue and red dot represents the averaged performance across all testing trials. The error bar represents two S.E.M across the mean. The transparent blue and red dots represent the model's performance on each testing trial for the phrase condition and the sentence condition, respectively. The results indicate no performance differences of the model between the phrase condition and the sentence condition. (c) The comparison between the real neural response (dashed lines) and the averaged model predicted response (solid blue for the phrase, solid red for the sentence) at a sample sensor Cz. The results show that both the averaged model for the phrase condition $\left(r=0.47^{* * *}, \mathrm{n}=1024\right)$ and the sentence condition ( $r=0.41$ (negative) on the time from 0 to $400 \mathrm{~ms}$ and on frequency between $0.1 \mathrm{kHz}$ to $0.8 \mathrm{kHz}$. The figure on the left and right side of

571 the upper panel represent the clustered STRF for the phrases at the left and right hemisphere, respectively. The corresponding

572 position of the lower panel represent the clustered model for the sentence condition. (e) The figure shows how the sensors

573 were selected, in which the bigger the red circle represents the more times the sensor was selected across all participants. (f)

574 The TRFs that were decomposed from the STRFs, in which the blue and red lines represent the phrase condition and the 
576 comparison of the magnitude of the TRFs, the blue and red bars represent the phrase condition and the sentence condition,

577 respectively. The error bar shows 1 s.e.m across the mean on each side. A 3-way repeated measure ANOVA of the peak

578 magnitude was conducted on the factors of stimulus-type (phrase or sentence), Hemisphere (left or right) and peak-type ( 100

$579 \mathrm{~ms}$ or $\sim 300 \mathrm{~ms})$. The results indicated a main effect of stimulus-type and a 3-way interaction. The post hoc comparison on the

580 main effect of stimulus-type suggested that the amplitude (negative) was stronger for the phrase condition than the sentence

581 condition (t (59) $=4.55, \mathrm{P}<2 \mathrm{e}-5^{* * *}$ ). To investigate the 3-way, Stimulus-type*Peak-type*Hemisphere, interaction, two 2-way repeated measure ANOVA with the Bonferroni correction were conducted on the factors of Hemisphere and Audio-type at each level of the Peak-type. The results indicated a main effect of stimulus-type at the first peak $(F(1,14)=8.19, p=0.012 *)$ and a 2-way Hemisphere*stimulus-type interaction at the second peak $(F(1,14)=6.42, p=0.023 *)$. At the first peak, a post hoc comparison on the main effect of stimulus-type was conducted using a paired sample t tests, the results showed that the magnitude of the phrase condition was higher than the magnitude of the sentence condition $(t(29)=3.49, p=0.001 * * *)$. For the 2-way, Hemisphere*stimulus-type, interaction at the second peak, the paired sample t tests with Bonferroni correction was conducted to compare the difference of the magnitude between the phrase condition and the sentence condition at each hemisphere. The results indicate that the magnitude at the second peak was stronger for the phrase condition than the sentence condition in the right hemisphere $\left(t(14)=3.21, p=0.006^{* *}\right)$, but not the left hemisphere $(t(14)=0.86, p=0.40)$. (h)

591 The comparison of the peak latency of TRFs, the blue and red bars represent the phrase condition and the sentence condition, respectively. The error bar shows 1 s.e.m across the mean on each side. A 3-way repeated measure ANOVA of the peak latency was conducted on the factors of stimulus-type (phrase or sentence), Hemisphere (left or right) and peak-type ( 100 ms or 300 ms). The results indicated a main effect of peak-type and a 3-way interaction. The post hoc comparison on the main effect of peak-type suggested that the latency of the first peak was significantly faster than the second peak $(t(59)=38.89, p<2 e-16$ $\left.{ }^{* * *}\right)$. The post hoc comparison on the 3-way interaction with the Bonderroni correction on the factors of Hemisphere and

597 stimulus-type for each level of the Peak-type suggested a 2-way Hemisphere*stimulus-type interaction at the first peak (F (1,

598 14) $\left.=12.83, p=0.002^{* * *}\right)$. The post hoc comparison on this 2 -way interaction using paired sample $t$ tests with the Bonferroni

599 correction indicated that the latency at the first peak was significantly longer for the sentences than the phrases at the right

600 hemisphere $\left(t(14)=3.55, p=0.003^{* * *}\right)$, but not the left hemisphere $(t(14)=0.58, p=0.56)$. (i) The SRFs which were

601 decomposed from the STRFs, in which the red and blue lines represent the phrase condition and the sentence condition,

602 respectively, the solid and the dashed lines represent the left and right hemisphere, respectively. (j) The comparison of the

603 amplitude of the SRFs. The SRF was first separated into three bands, low ( $<0.1 \mathrm{kHz})$, middle ( 0.1 to $0.8 \mathrm{kHz})$ and high ( $>0.8$ 


\section{Methods}

\section{Participants.}

Fifteen right-handed Dutch native speakers, 22-35 years old, 7 males, participated in the

621 participant before the experiment.

\section{Stimuli.}

623 We selected fifty line-drawings of common objects from a standardized corpus

624 (Snodgrass \& Vanderwart, 1980). The Dutch names of all objects were monosyllabic and had

625 non-neuter lexical gender. In our experiment, the objects appeared as colored line-drawing on 
626

627

628

629

630

631

632

633

634

635

636

637

638

639

640

641

\section{Experimental Procedure.}

643

644

645

646 to $2.29^{\circ}$ of visual angle for the participant.

a grey background. More specifically, we presented each line-drawing in five colors: blue (blauw), red (rood) yellow (geel), green (groen) and purple (paars). In total, this yielded 250 pictures. The line-drawings were sized to fit into a virtual frame of $4 \mathrm{~cm}$ by $4 \mathrm{~cm}$, corresponding

We then selected ten figures with different objects in each color, without object replacement between colors, to create speech stimuli. For each selected line-drawing, a 4syllable, phrase-sentence pair was created, i.e. De rode vaas (The red vase) and De vaas is rood (The vase is red). In total, we had 100 speech stimuli (50 phrases and 50 sentences). All stimuli were synthesized by a Dutch male voice, "Guus", of an online synthesizer (www.readspeaker.com). All speech stimuli were 733 ms to 1125 ms in duration (Mean= 839 $\mathrm{ms}, \mathrm{SD}=65 \mathrm{~ms})$. In order to normalize the synthesized auditory stimuli, they were first resampled to $44.1 \mathrm{kHz}$. Then all speech stimuli were adjusted by truncation or zero padding at both ends to 1000 ms without missing any meaningful dynamics. Then $10 \%$ at both ends of each stimulus was smoothed by a linear ramp (a sine wave) for removing the abrupt sound burst. Finally, for normalizing the intensity of speech stimuli, the root mean square (RMS) value of each stimulus was normalized to $-16 \mathrm{~dB}$.

Each trial started by a fixation cross at the screen center (500 ms in duration).

Participants were asked to look at the fixation. Immediately after the fixation cross had disappeared, the participants heard a 1000 ms long spoken stimulus, either a phrase or a sentence, followed by three-second silence; then the participants were asked to perform one of 
647 three types of task, indicated to them by an index (1, 2 or 3 showing at the screen center, 500 ms in duration). If the index was ' 1 ', they did a linguistic structure discrimination task (type-one task), in which they had to judge whether the spoken stimulus was a phrase or a sentence and 650 indicate their judgment by pressing a button. If the index was ' 2 ', a picture would follow (200 651 ms in duration) and would be shown after a 1000 ms gap after the index number. Then 652 participants would do a color matching task (type-two task), in which they had to judge whether the color described in the spoken stimulus matches the color of the shown picture and

654 indicate their judgment by pressing a button. If the index is ' 3 ', they would experience the same procedure as the type two task, except for they would do an object matching task (type-three

657 indicating their respond by pressing a button. All responses were recorded via a parallel port response box, in which each one of the two buttons were represented as phrase/match and sentence/mismatch, respectively. Each response was followed by a silent interval of 3 to 5.2 seconds (jittered). The experimental procedure is depicted in the Fig $\mathbf{8}$.

Fig 8. Experimental procedure. An illustration of the experimental procedure. Participants were asked to looking at the screen center, after hearing the speech stimulus they would do a task indexed by a number that showed on the screen. If the number was ' 1 ', they would judge whether the heard stimulus was a phrase or a sentence. If the number was ' 2 ', they would see a 
671 data collection, several practice trials were conducted for each participant in order to make

672 sure they had understood the task. Trials in each block were fully matched in across linguistic-

673 structure (phrase or sentence) and task-type (type 1, type 2 or type 3). For instance, half of the

674 spoken stimuli were phrases and half were sentences (twenty-four in each type), six types of

675 combinations (8 trials for each type) were evenly distributed in each block $(8 * 2 * 3)$ etc. Trial

676 order was pseudo random throughout the whole experiment. The behavioral results indicated

677 the task was relative easy and no difference between phrase and sentences. All tasks combined

678 accuracy for phrases and sentences were $97.9 \pm 3 \%$ and $97.3 \pm 3 \%(p=0.30)$, respectively.

679 After the main experiment, a localizer task was performed, in which a tone beep $(1 \mathrm{kHz}, 50 \mathrm{~ms}$

680 in duration) was played 100 times (jitter 2 to 3 seconds) for each participants to localize the

681 canonical auditory response (N1-P2 complex). The topographies for N1 and P2 are shown in Fig

682 9. The upper panel shows the averaged N1-P2 complex of all participants over the time bin

683 from 90 - 110 ms for the N1 and $190-210$ ms for the P2. The lower panel shows the N1-P2

684 complex after the Surface Laplacian (Cohen, 2014; Perrin, Bertrand, \& Pernier, 1987; Perrin,

685 Pernier, Bertnard, Giard, \& Echallier, 1987), in which the effect of the volume conduction was

686 attenuated. The topographies indicated that all participants had the canonical auditory

687 response.

688 Fig 9. Auditory N1-P2 complex. The topographical distribution of the canonical auditory N1-P2 complex. The upper 
692

693

694

695

696

697

698

699

700

701

702

703

704

705

706

707

708

709

710

711

712

\section{EEG recording}

We recorded EEG data using a 64-channel active sensors system of Brain Products (Brain Products $\mathrm{GmbH}$ ) in a sound dampened, electrically shielded room. Signals were digitized online at $1000 \mathrm{~Hz}$, high-pass and low-pass at $0.01 \mathrm{~Hz}$ and $249 \mathrm{~Hz}$, respectively. Two electrodes, AFz and $\mathrm{FCz}$, were used as ground and reference. All electrodes were placed on the scalp based on the international 10-20 system and the impedance of each one was kept below $5 \mathrm{k} \Omega$.

We presented stimuli using MATLAB 2019a (The MathWorks, Natick, MA) with the Psychtoolbox-3 (Brainard, 1997). Auditory stimuli were played at $65 \mathrm{~dB}$ SPL and delivered through air tubes ear plugs (Etymotic ER-3C, Etymotic Research, Inc.). Event markers were sent via a parallel port for tagging the onset of the interested events (i.e., speech onset, task index onset, etc.).

\section{EEG data preprocessing}

We preprocessed EEG data via MATLAB using EEGLAB toolbox (Delorme \& Makeig, 2004) and customized scripts. We first down-sampled the data to $256 \mathrm{~Hz}$, then high-pass filtered at $0.5 \mathrm{~Hz}$ (finite impulse response filter, FIR; zero-phase lag), and cleaned by the Artifact Subspace Reconstruction (ASR) (Chang, Hsu, Pion-Tonachini, \& Jung, 2018; Kothe \& Jung, 2016). We interpolated all detected bad channels with spherical interpolation. After transfer data to average reference, the online reference $\mathrm{FCz}$ was recovered and the line noise, $50 \mathrm{~Hz}$ and its harmonics, was removed.

Following the above mentioned steps, we extracted epochs of $2 \mathrm{~s}$ preceding and $9 \mathrm{~s}$ after auditory stimulus onset. We conducted deletion of bad trials deletion and artifacts in the 
713 following two steps. First, we used independent component analysis (ICA) for decomposing

714 data to component space (number of components equals data rank). Then for each

715 independent component, we used short-time Fourier Transform (STFT) to convert each trial

716 into power spectrum, in which we extracted a value which was calculated by the power

717 summation between $15 \mathrm{~Hz}$ to $50 \mathrm{~Hz}$. Then all the extracted values, one value per epoch, in each

718 component formed a distribution. From this distribution, we transformed all the extracted

719 values to $z$-score, the epochs with the value outside the range of plus and minus three standard

720 deviation were deleted. Second, ICA was conducted again on the trial-rejected data for eye-

721 related artifacts removal and muscular activities elimination. Artifact components were

722 identified and removed by an automatic classification algorithm (Winkler, Haufe, \&

723 Tangermann, 2011). All the preprocessing steps resulted in the removal of, on average, 7

724 components (range 4 to 11 ) and 22 trials (including incorrect trials and trials with too slow

725 response, range 10 to $30,4 \%$ to $12.5 \%$ ) per participant. Finally, volume conduction was

726 attenuated by applying the Surface Laplacian (Cohen, 2014; Perrin et al., 1987; Perrin et al., 727 1987).

728 EEG data analysis

\section{Time Frequency Decomposition}

We convolved the single-trial time series with a family of complex wavelets ( 1 to $50 \mathrm{~Hz}$

731 in 70 logarithmically spaced steps), optimizing temporal and spectral resolution by changing the

732 cycle from 3 to 30 in logarithmical steps. 
We calculated phase coherence by inter-trial phase clustering (ITPC) ( Cohen, 2014; coefficients of all trials were divided by their corresponding magnitude and averaged across trials. The magnitude of the averaged complex output was represented as phase coherence 737 (ITPC).

The induced neural response (power) was extracted from the analytical output at each time-frequency bin by taking the summation of the squared wavelet coefficients. Decibel

740 transformation was performed separately at each frequency, in which the average power at the 741 duration from 800 ms to 200 ms before the audio onset was used as the baseline.

\section{Phase Connectivity}

We first decomposed trials in each condition via wavelet convolution (same parameters as the time-frequency decomposition). Then, we calculated the Cross Spectral Density (CSD) for each sensor-pair at each frequency-time-trial bin. We calculated phase connectivity over the sensor space by Inter Site Phase Coherence (ISPC) (Cohen, 2014; Lachaux et al., 2000; Lachaux

747 et al., 1999; Mormann et al., 2000), in which we divided the complex coefficients by the corresponding amplitude at each frequency-time-trial bin then computed averages across all

750 between sensors (ISPC). After the above mentioned steps, the phase connectivity at each time-

751 frequency bin was represented as an all-sensor to all-sensor matrix, in our case, 65 sensors * 65 752 sensors. 
To transform the connectivity matrix to connectivity degree at each time-frequency bin,

we formed a distribution by pooling together all the connectivity values from both conditions,

then defined the threshold as the value which is half a standard deviation above the median.

We then binarized the connectivity matrices for both conditions by applying this threshold at

each bin. The connectivity degree at each time-frequency bin was then represented as the

count of the number of the connectivity values that above this threshold. Finally, we

normalized the connectivity level at each time-frequency bin to percentage change relative to

ms to 200 ms before the audio onset.

\section{Phase Amplitude Coupling (PAC)}

Since low-frequency phase and high-frequency amplitude may show coupling during

773 vectors in the complex time series (Canolty et al., 2006; Cohen, 2014). Since the variation of the 
775 More specifically, after calculating the real PAC value using the raw complex time series, the

776 random PAC value were computed 1000 times using the re-constructed complex time series,

777 which were built by temporally shifting the amplitude time series with a random temporal

778 offset. These 1000 random PAC values formed a reference distribution for each phase-

779 amplitude bin. Then the $z$-score of the real PAC value in this distribution was represented as the 780 index of the phase-amplitude coupling, PAC-Z.

781

782

783

784

785

786

787

788

\section{Power Connectivity}

After time-frequency decomposition, we extracted induced power at each channel-

time-frequency-trial bin. For each condition, we calculated power connectivity for each sensor-

pair at each time-frequency bin as the Rank Correlation between the power response of all

trials in one sensor and the power response of all trials in the other sensor (Bruns, Eckhorn,

Jokeit, \& Ebner, 2000; Cohen, 2014; Hipp, Hawellek, Corbetta, Siegel, \& Engel, 2012). The power

connectivity calculation resulted in an all-sensors to all-sensors (65*65 in our case)

representation at each time-frequency bin for each condition.

To transfer the power connectivity at each time-frequency bin to the power connectivity degree, we applied a statistical thresholding method. More specifically, at each time-frequency bin, we formed a distribution by pooling together all the connectivity values from both conditions, then defined the threshold as the value which is half a standard deviation above the median. We then binarized the connectivity matrix at each bin for each condition by applying the corresponding threshold. The connectivity degree at each time-frequency bin was represented as the count of the number of the connectivity values that above this threshold.

Finally, we transferred the connectivity level at each time-frequency bin as a percentage change 
797 relative to the connectivity level of the baseline which was calculated as the average connectivity level in the duration from $800 \mathrm{~ms}$ to $200 \mathrm{~ms}$ before the audio onset.

799

800

801

802

803

804

805

806

807

808

\section{Spectral Temporal Response Function (STRF)}

The STRF is a linear kernel which convolves with the specified features of the speech signal to estimate the neural response in time. It can be interpreted as a linear filter which transforms the stimulus feature to the neural response (Crosse, Di Liberto, Bednar, \& Lalor, 2016; Di Liberto et al., 2015).

In our study, the stimulus feature (SF) was defined as the spectrogram, which was obtained by filtering the speech stimulus into 16 logarithmically spaced frequency bands between 0.05 to $8 \mathrm{kHz}$ for mimicking the frequency decomposition by the brain (Greenwood, 1990). The temporal envelope (energy profile) for each frequency band was then extracted by Hilbert Transform.

To construct the stimulus-response pairs, we first applied a linear ramp to both sides of each trial corresponded neural response ( $10 \%$ at each side) to attenuate the abrupt onset and offset. Then, we matched each 1-second neural response with the corresponding SF.

In order to optimize the estimation of the STRF, a randomization procedure was applied to create new data structure. We first randomly selected $80 \%$ of all unique speech stimuli, then the stimulus-response pairs that corresponded to the selected speech stimuli were extracted as the seed data to construct the training dataset for performing the cross validation. We constructed thirty five 10-second long stimulus-response pairs, in which each one of them was a concatenation of the randomly selected ten 1-second stimulus-response pair (Bootstrapping). 
The STRF was estimated using the Ridge Regression with leave-one-out cross validation.

STRF. The model with the Lambda parameter which gave the best performance was used as the optimized STRF.

835 procedure that mentioned above. In order to check the performance of each estimated STRF,

836 the stimulus-response pairs (the unseen pairs for each STRF) that corresponded to the

837 remaining $20 \%$ of the speech stimuli were extracted as the seed data for constructing the

838 testing dataset. We extracted five 4-second long stimulus-response pairs in the testing data for 
839

840

841

842

843

844

845

846

847

848

849

850

851

852

853

854 Function Toolbox (Crosse et al., 2016).

855 and at the same time takes into account the dependencies (temporal, spatial and spectral

each STRF, each one of them was a concatenation of randomly selected four 1-second long pairs.

The real performance of each STRF was calculated by using the frequency and condition matched stimulus-response pairs. The random performance was calculated 1000 times using the pairs which were constructed by combining the stimulus with a randomly selected neural response.

For fitting the low frequency STRF $(<9 \mathrm{~Hz})$, the same procedure was conducted. The performance of the averaged STRF in each condition was computed using the averaged

Pearson's correlation between the real neural response and the predicted response across sensors.

We extracted the temporal response function (TRF) and the spectral response function (SRF) for each participant at each condition by averaging the STRF over the frequencies from $0.1 \mathrm{kHz}$ to $800 \mathrm{kHz}$ and by averaging the STRF over the time from 0 to $400 \mathrm{~ms}$, respectively.

All the calculations in this section were conducted using customized scripts, the scripts of EEGLAB toolbox (Delorme \& Makeig, 2004), and the Multivariate Temporal Response

\section{Statistical Analysis}

In addition to using parametric statistical methods, we applied a cluster-based nonparametric permutation test. This method deals with the multiple-comparisons problem adjacency) in the data. For all types of analysis that followed this inference method, we initially 
860

861

862

averaged the subject-level data over trials and for each single sample, i.e. a time-frequency-

channel point, and performed a dependent t-test. We selected all samples for which the t-value exceeded an a priori threshold, $p<0.05$, two-sided, and subsequently clustered these on the basis of spatial and temporal-spectral adjacency. The sum of the t-values within a cluster was used as cluster-level statistic. The cluster with the maximum sum was subsequently used as the test statistic. By randomizing the data across the two conditions and recalculating the test statistic 1000 times, we obtained a reference distribution of the maximum cluster $t$ values. This distribution was used to evaluate the statistical distribution of the actual data. This statistical method was implemented using the FieldTrip toolbox (Maris \& Oostenveld, 2007; Oostenveld, Fries, Maris, \& Schoffelen, 2011).

\section{Acoustic analysis}

The intensity fluctuation of each speech stimulus was characterized by the corresponding temporal envelope, which was extracted by the Hilbert Transform of the halfwave rectified speech signal. Then each extracted temporal envelope was downsampled to 200 $\mathrm{Hz}$ and the Discrete Fourier Transform (DFT) was performed to extract the spectrum. Decibel transformation for the spectrum of each speech stimulus was performed by using the highest frequency response in the corresponding phrase-sentence pair as the reference. Fig 10a shows the comparison between the averaged spectrum of all phrases and the averaged spectrum of all sentences. The shaded area covers 2 standed errors of the mean across the stimuli. Statistical comparison (the paired-sample t-test) was conducted at each frequency bin, in which no evidence was found to indicate significant physical difference between phrases and sentences. Fig 10b shows the acoustic features of a phrase-sentence pair, De rode vaas (The red 
vase) and De vaas is rood (The vase is red), the upper-left panel shows the comparison of the spectrogram the sample phrase and the sample sentence, respectivily.

Fig 10. Stimuli comparison. (a) Spectrum comparison between the two types of speech stimuli. The shaded area for envelopes of the sample phrase-sentence pair, i.e., De rode vass (the red vase) v.s. De vaas is rood (the vase is red), that down sampled to $200 \mathrm{~Hz}$. The lower-left panel shows the spectrum for the sample phrase-sentence pair, in which the horizontal axis and the vertical axis shows the frequency response of the temporal envelop of the phrase and the sentence, respectively. The Pearson's correlation indicated that the spectrum are highly similar between the sample phrase and the sample sentence $(r=$ $\left.0.94, p<1 e-5^{* * *}\right)$. The upper-right and lower-right panel shows the spectrogram of the sample phrase and the sample sentence, respectively.

\section{Discussion}

900 In this study, we investigated the neural responses to minimally different linguistic structures by

901 minimizing their differences in acoustic-energetic/temporal-spectral profiles and semantic 
904 the dimesion of neural readouts that were sensitive to the distinctive linguistic structures

905 between phrases and sentences. We asked whether phrases and sentences have different

906 effects on functional connectivity, and found, first, that while phrases and sentences recruit

907 similar functional networks, the engagement of those networks scaled with linguistic structure.

908 Sentences showed more phase coherence and power connectivity compared to phrases. This

909 connectivity pattern suggests that phrases and sentences differently impact the distribution

910 and intensity of neural networks involved in speech comprehension. Second, we found that

911 phase-amplitude coupling between theta and gamma, which has been implicated in speech

912 processing, is not sensitive to structural differences in spoken language. Third, we found that

913 activity in the alpha band was sensitive to linguistic structure. Lastly, by modelling acoustic

914 fluctuations in the stimulus and brain response with STRFs, we found that phrases and

915 sentences differentially relied on the acoustic response in the brain, and that sentences were

916 more abstracted away from acoustic dynamics in the brain response. In the following sections

917 we give more detail about our findings and discuss potential interpretations of them.

919 Phase coherence

920 Consistent with previous research (Doelling et al., 2014; Luo \& Poeppel, 2007; Peelle \&

921 Davis, 2012; Peelle et al., 2013), our phase synchronization analysis detected low frequency

922 phase coherence during speech comprehension. Moreover, phase coherence distinguished

923 between phrases and sentences, yielding a cluster between $\sim 450$ and $\sim 900 \mathrm{~ms}$ after audio

924 onset and from $\sim 2 \mathrm{~Hz}$ to $\sim 8 \mathrm{~Hz}$ in frequency, that was most pronounced over central electrodes. 
925 These results therefore suggest that syntactic structure may be encoded by low frequency

926 phase coherence, through systematic organization of activity in neural networks, in particular

927 their temporal dynamics. Our results are consistent with the notion of phase sets in

928 computational models of structured representations that exploit oscillatory dynamics. Phase

929 sets are representational groupings that are formed by treating distributed patterns of

930 activation as a set when units are in (or out) of phase with one another across the network

931 (Doumas \& Martin, 2018; Martin \& Doumas, 2017, 2019, 2020). They are key to the

932 representation of structure in artificial neural network models.

934 Phase connectivity

935 Phrases and sentences also yielded differences in phase connectivity. In the predefined

936 time and frequency range of interest, the statistical comparison indicated a difference

937 corresponding to a cluster approximatly from 800 to 1600 ms after the audio offset,

938 occurring at the very low frequency range $(<\sim 2 \mathrm{~Hz})$ that was most pronounced over the right

939 posterior region. Phrases and sentences thus differentially impact the temporal synchronization

940 of neural responses.

942 suggests that the effect on temporal synchronization occurs after the initial presentation the

943 speech stimulus. In our experiment, participants were randomly presented a task prompt for

944 three possible tasks (color discrimination, object discrimination, phrase or sentence

945 discrimination), which asked them to determine either 'semantic' (object or color information) 
or 'syntactic' information (whether the stimulus was a phrase or sentence) from the speech

the task prompt. The tasks also insured that participants could not select a single dimension of

the stimulus for processing. Similarly, because we used an object and a color task, participants

had to distribute their attention evenly across the adjectives and nouns, mitigating word order between them. previous research (Brennan \& Martin, 2020; Ding et al., 2016; Kaufeld et al., 2020; Keitel et al., timing of the occurrence of linguistic structure; low frequency neural responses $(1-2 \mathrm{~Hz})$ were

960 found to track the highest level linguistic structures (phrases and sentences) in their stimuli.

961 Here we extended their work to ask whether the $1 \mathrm{~Hz}$ response could be decomposed to reflect separate syntactic structures (phrases vs. sentences), and we identified the role of phase in except for the presence of syntactic structure, the stimuli were normalized to be highly similar. 
Lastly, phrases and sentences differed most strongly over right-posterior parietal cortex,

which is broadly consistent with previous research on speech comprehension. Functional

Magnetic Resonance Imaging (fMRI) studies implicate the posterior right hemisphere in

processing syntactic structure (de Bode, Smets, Mathern, \& Dubinsky, 2015; Grodzinsky, 2000;

972

Grodzinsky \& Friederici, 2006; Maess, Koelsch, Gunter, \& Friederici, 2001). Neurophysiological

973

research also suggests the involvement of right hemisphere in extraction of slow timescale

974 information extraction (Abrams, Nicol, Zecker, \& Kraus, 2008; Giraud et al., 2007; Morillon,

975 Liégeois-Chauvel, Arnal, Bénar, \& Giraud, 2012; Poeppel, 2003). In addition, the P600, a positive

976 ERP component often associated with syntactic processing, has a robust right posterior

977 topographical distribution (Coulson, King, \& Kutas, 1998; Friederici, Pfeifer, \& Hahne, 1993;

978 Hagoort, Brown, \& Groothusen, 1993; Osterhout \& Holcomb, 1992; Osterhout \& Mobley, 1995;

979 Patel, Gibson, Ratner, Besson, \& Holcomb, 1998). In light of the existing literature, therefore,

980 the right-posterior distribution of the phase connectivity effects is consistent with processing of

981 syntactic structures, although we refrain from claims about underlying neural sources based on

our EEG data.

\section{Phase Amplitude Coupling (PAC)}

We observed PAC during speech comprehension, as a low frequency phase response ( 4-10 Hz) entrained with high frequency amplitude ( $15-40 \mathrm{~Hz})$. This effect appeared largely over the bilateral central area. The bilaterial central topographical distribution has been replicated shown reflecting sensory-motor integration (Babiloni et al., 2011; Klimesch, Sauseng, \& HansImayr, 2007; Neuper, Wörtz, \& Pfurtscheller, 2006; Pfurtscheller, Brunner, Schlögl, \& Da Silva, 2006; Pfurtscheller, Neuper, Schlogl, \& Lugger, 1998; Schlögl, Lee, Bischof, \& Pfurtscheller, 
2005; Suffczynski, Kalitzin, Pfurtscheller, \& Da Silva, 2001), which is consistent with the proposal from Giraud and Poeppel (2012) that PAC reflects an early step in speech encoding involving sensory-motor alignment between the auditory and articulatory systems. Crucially, however, this effect did not distinguish phrases and sentences. Although a null result, this pattern is compatible with the generalized model for speech perception (Giraud \& Poeppel, 2012). This early step is presumably similar for phrases and sentences, and perhaps for any type of structure above the syllable level.

\section{Induced alpha power}

Induced alpha band power distinguished phrases and sentences, and this effect was most pronounced at the left hemisphere. This pattern suggests involvement of alpha band oscillations in syntactic structure processing. Although alpha band activity is often associated with attentional or working memory related processing (Haegens et al., 2010; Obleser et al., 2012; Strauß et al., 2014; Ten Oever et al., 2020; Wilsch \& Obleser, 2016; Wöstmann et al., 2016; Wöstmann et al., 2015; Wöstmann et al., 2017), we do not consider this a very plausible alternative explanation for our results. For example, it not clear why phrases and sentences would differ in their attentional demands. In addition, we employed an experimental task to ensure similar attention to phrases and sentences, and phrases and sentences were associated with similar behavioral performance in each task (with a caveat that performance was at ceiling and may therefore not pick up on small differences between conditions).

We do not claim that that all speech-elicited alpha band effects reflect syntactic processing. Some observed effects effects may well reflect perceptual processing during speech 
1011 comprehension (e.g. Obleser \& Weisz, 2012), especially in experiments designed to manipulate

1012 perceptual processing, such as speech-in-noise manipulations. Neural response in a given band,

1013 e.g., the alpha band, need not reflect only a one particular perceptual process. Likewise, the

1014 fact that the alpha band neural response could reflect lower-level perceptual processes or

1015 working memory load in certain contexts does not necessarily rule out its role in higher-level

1016 linguistic information representation, e.g., syntactic information.

\section{Power connectivity}

Phrases and sentences elicit differences in induced power connectivity in alpha band activity (7.5 to $13.5 \mathrm{~Hz}$ ). Phrases showed more inhibition in power connectivity than sentences;

1021 in other words, sentences showed a stronger connectivity degree over sensor space in the

1022 alpha band than phrases. Several aspects of these results are noteworthy. First, we observed

1023 this effect from 100 ms until 2200 ms after stimulus onset, which suggests the effect in

1024 functional connectivity persisted rather long and outlasted the observed effects in induced

1025 alpha power, which we observed from 350 ms to 1000 ms after the audio onset (during the

1026 listening stage). The extended nature of the functional connectivity effect could reflect the

1027 continuing integration and representation of syntactic and semantic components.

1030 in time and 7.5 to $13.5 \mathrm{~Hz}$ in frequency). Whereas phrase and sentences showed similar

1031 functional connectivity in the intensity of the neural response, sentences showed stronger 
1032 inter-region (sensor) connectivity than phrases. By design, in our stimuli sentences had more

1033 constituents than phrases did. If local network activity is more organized or coherent as a

1034 function of linguistic structure, then the difference observed here could reflect the encoding of

1035 additional constituents in sentences compared to phrases.

Lastly, phrases elicit stronger inhibition of induced power connectivity than sentences

did. This indicates weaker cooperation between brain regions, in other words, regions showing more independent the neural response. In contrast, inter-region connectivity was stronger for

1039 the sentence condition than the phrase condition, which suggested a higher-level of intensity of

1040 connectivity between brain regions for the sentences in order to separate them from the 1041 phrases.

In sum, phrase and sentences elicited robust differences in induced power connectivity.

1043 A similar functional connectivity pattern was deployed for representing phrases and sentences,

1044 but the intensity of the connectivity was stronger for sentences than phrases. This finding is

1045 consistent with the prediction that low frequency power and network ogranization should

1046 increase as linguistic structure increases. Our stimuli were designed to allow the measurement

1047 of differences in neural dynamics between phrases and sentences, and as such differed in the

1048 number and type of linguistic constituents that were perceived. Beyond the number and type of

1049 consituents, the phrase and sentence structures also differ in the relations between

1050 constiuents, or in the linguistic notion of hierarchy. But given the co-extension of number, type,

1051 and relation, our stimuli and design do not yet allow us to determine if, nor how, these

1052 variables alone might affect structural encoding in neural dynamics. 
1054 Spectrotemporal response functions (STRF)

1055

1056

1057

1058

1059

1060

1061

1062

1063

1064

1065

1066

1067

1068

1069

1070

1071

1072

1073

1074

We performed STRF analysis to investigate whether phrases and sentences are encoded differently. Firstly, consistent with previous research (Ding \& Simon, 2012a, 2012b), only low frequency $(<9 \mathrm{~Hz})$ neural responses robustly reflected the phase-locked encoding of the acoustic features. Secondly, we observed a bilaterial representation of the slow temporal modulations of speech, in particular at posterior sensors. The posterior region has consistently been found to be involved in syntactic integration (Coulson et al., 1998; Friederici et al., 1993; Hagoort et al., 1993; Osterhout \& Holcomb, 1992; Osterhout \& Mobley, 1995; Patel et al., 1998). The low frequency neural response that models the phase-locked encoding of acoustic features can capture structural differences between phrases and sentences, even without explicitly using a hand-coded annotation on the syntactic level to reconstruct the data. Thirdly, and most importantly, we explored these patterns further in both the temporal and spectral dimension, by decomposing the STRF into the TRF and the SRF. Both TRF and SRF suggested a different encoding mechanism between phrases and sentences. More specifically, the TRF results showed that the brain transduces the speech stimulus into the low frequency neural response via an encoding mechanism with two peaks in time (at $\sim 100 \mathrm{~ms}$ and $\sim 300 \mathrm{~ms}$ ). The two peaks reflect the instantaneous low frequency neural response that is predominantly driven by the encoding of acoustic features that were presented $\sim 100$ ms and $~ 300$ ms ago. In the two time windows that centered at $\sim 100 \mathrm{~ms}$ and $\sim 300 \mathrm{~ms}$, respectively, phrases and sentences showed a different dependency on the acoustic features in both latency and intensity. 
When we only consider intensity ( $100 \mathrm{~ms}$ time window), sentences depended on

1076

1077

1078

1079

1080

1081

1082

1083

1084

1085

1086

1087

1088

1089

1090

1091

1092

1093

1094

1095

1096

acoustic features less strongly than phrases. This result is consistent with the idea that sentence representations are more abstracted away from the physical input because they contain more linguistic structural units (i.e., constituents) that are not verdically present in the physical or sensory stimulus. Consistent with previous research, we found that the instantaneous neural response was strongly driven by the encoding of the acoustic features presented $\sim 100 \mathrm{~ms}$ ago (Brodbeck et al., 2018; Crosse \& Lalor, 2014; Di Liberto et al., 2015; Ding \& Simon, 2012a, 2012b, 2013; Golumbic et al., 2013; Puvvada \& Simon, 2017; Wang, Zhang, Zou, Luo, \& Ding, 2019).

When we only consider the latency ( 100 ms time window), and only at the right hemisphere, the low frequency neural response of the sentences was predominantly driven by the acoustic features that appeared earlier in time than the acoustic features that drove the neural response to phrases. Our results imply that the brain distinguishes syntactically different linguistic structures by how driven its responses are by the acoustic features that appeared at 100 ms ago. More importantly, at the right hemisphere, the findings suggested that the low frequency neural response of the sentences reflected the encoding of the acoustic features that appeared earlier in time than the acoustic features that drove the neural response of the phrases. This could be evidence that the right hemisphere is dominant in extracting the slow timescale information of speech that is relevant for, or even shapes, higher-level linguistic structure processing, e.g., syntactic structure building (Ding \& Simon, 2012a, 2012b; Poeppel, 2003). It is noteworthy to see that the distribution in time and space of these patterns is consistent with the idea that the brain is extracting information from the sensory input at 
1097 different timescales, and that this process is in turn is reflected in the degree of departure (in

1098 terms of informational similarity) of the neural response from physical features of the sensory

1099 input.

At $\sim 300 \mathrm{~ms}$, when we only consider intensity, the low frequency neural response to

1101 phrases is more strongly dependent on the acoustic features than the neural response to

1102 sentences is, showing again that sentences are more abstracted from sensory representations.

1103 However, the comparison of the TRF indicated that the brain exploited a different encoding

1104 mechanism at the right hemisphere between the phrases and the sentences. More concretely,

1105 the low frequency neural response of the phrases showed a stronger dependency on the

1106 acoustic features than the sentences at the right hemisphere, but not at the left hemisphere.

1107 The results, first, suggested that the instantaneous low frequency neural response reflects the

1108 encoding of the acoustic features that were present $\sim 300 \mathrm{~ms}$ ago, in both conditions. Then, it

1109 reflected that, only in the right hemisphere, the low frequency neural response of the phrases

1110 more strongly depends on the acoustic features from $\sim 300$ ms ago when compared to the

1111 neural response to sentences. This finding reminds us of the results of the phase connectivity

1112 analysis, in which the phase connectivity degree also showed a different pattern between the

1113 phrases and the sentences at the right posterior region; which is consistent with previous

1114 research that has shown a role for the right hemisphere in processing slow modulations in

1115 speech, such as prosody (Abrams et al., 2008; Ding \& Simon, 2012a, 2012b; Giraud et al., 2007;

1116 Kerlin, Shahin, \& Miller, 2010; Luo \& Poeppel, 2007; Poeppel, 2003). Consistent with these

1117 findings, our results further indicated that the brain can separate syntactically different

1118 linguistic structures via differential reliance by the right hemisphere on representations of the 
1119 acoustic features that appeared at $300 \mathrm{~ms}$ ago. That sentence representations were more

1120 abstract and less driven by the acoustics in the left hemisphere is consistent with contemporary

1121 neurobiological models of sentence processing (Friederici, 1995; Hagoort, 2013), although we

1122 cannot attribute the differences we observed to a single aspect of linguistic structural

1123 descriptions (i.e., to number, type, relations between constituents, or whether constituents

1124 form a 'saturated' sentence or not).

1126 differential reliance on the encoding of the acoustic features from roughly the first formant

1127 (Catford, 1988; Jeans, 1968; Titze et al., 2015; Titze \& Martin, 1998), and in a phase-locked

1128 manner. More specifically, in the range of the first formant, the low frequency neural response

1129 reflected a stronger dependency on the acoustic features in the phrases than in the sentences.

1130 Unlike consonants, the intensity of vowels are well reflected at the first formant $(<1 \mathrm{kHz})$

1131 (Catford, 1988; Jeans, 1968; Titze et al., 2015; Titze \& Martin, 1998). Although the overall

1132 physical intensity of the speech stimulus of the phrases was not different from the sentences,

1133 the neural response contains information that discriminates the differences between syntactic

1134 structures. Given that the stimuli were not physically different, this pattern of results strongly

1135 suggests that the brain is "adding" information, for example by actively selecting and

1136 representing linguistic structures that are cued by the physical input and its sensory correlate.

1137 For example, the brain could be adding phonemic level information, e.g., vowels, lexical, and

1138 phrasal via a top-down mechanism; in certain situations and languages, even a single vowel can

1139 cue differential syntactic structure. In fact, in our stimuli, the schwa carries agreement

1140 information that indicates the phrasal relationship between "red"(roode) and "vase" (vaas) in 
1141 the phrase "de roode vaas." Our results, which feature both dependence on, but also departure

1142 from, the acoustic signal, are consistent with previous findings that have shown low frequency

1143 cortical entrainment to speech and argued that is can reflect phoneme-level processing (Di

1144 Liberto \& Lalor, 2017; Di Liberto et al., 2015; Keitel et al., 2018; Khalighinejad, da Silva, \&

1145 Mesgarani, 2017). We extend these findings by showing that when lower-level variables in the

1146 stimuli are modeled, the brain response can discriminate between syntactic structures even

1147 without the addition of higher-level linguistic annotations in our TRF models, and that this

1148 result indicates that the degree of departure from the physical stimulus increased as abstract

1149 structure accrues.

\section{Summary}

1151 In this study, we found a neural differentiation between spoken phrases and sentences

1152 that were physically and semantically similar. Moreover, we found that this differentiation was

1153 captured in several readouts, or dimensions of brain activity (viz., phase synchroniziation,

1154 functional connectivity in phase and induced power). By modeling the phase-locked encoding of

1155 the acoustic features, we further show that the brain can represent the syntactic difference

1156 between phrases and sentences in the low frequency neural response, but that the more

1157 structured a stimulus is, the more it departs from the acoustically-driven neural response to the

1158 stimulus, even when the physicality of the stimulus is held constant. Across all our results, we

1159 provide a comprehensive electroencephalographic picture of how the brain separates linguistic

1160 structures within its representational repetoire. However, further research is needed to explore

1161 the relationship between these different neural readouts that indexed syntactic differences, 
bioRxiv preprint doi: https://doi.org/10.1101/2021.07.09.451747; this version posted July 9, 2021. The copyright holder for this preprint (which was not certified by peer review) is the author/funder, who has granted bioRxiv a license to display the preprint in perpetuity. It is made available under aCC-BY 4.0 International license.

1162 e.g., how the induced neural response at alpha band interacts with phase coherence in the low

1163 frequency $(<8 \mathrm{~Hz})$, and how these separation effects are represented at the neural source level. 


\section{Acknowledgments}

1166 AEM was supported by the Netherlands Organization for Scientific Research (NWO; grant

1167 016.Vidi.188.029), and a Max Planck Research Group and a Lise Meitner Research Group

1168 "Language and Computation in Neural Systems" from the Max Planck Society. The authors

1169 thank Mante S. Nieuwland for comments on an earlier version of this work, Shunag Bi for

1170 selecting stimuli and arranging figures, and Cas W. Coopmans for guidance as to visual

1171 representation of linguistic constituents.

1172

1173 


\section{Reference}

Abrams, D. A., Nicol, T., Zecker, S., \& Kraus, N. (2008). Right-hemisphere auditory cortex is dominant for coding syllable patterns in speech. Journal of Neuroscience, 28(15), 3958-3965.

Babiloni, C., Infarinato, F., Marzano, N., lacoboni, M., Dassù, F., Soricelli, A., . . Del Percio, C. (2011). Intra-hemispheric functional coupling of alpha rhythms is related to golfer's performance: A coherence EEG study. International Journal of Psychophysiology, 82(3), 260-268.

Becker, R., Pefkou, M., Michel, C. M., \& Hervais-Adelman, A. G. (2013). Left temporal alpha-band activity reflects single word intelligibility. Frontiers in systems neuroscience, 7, 121.

Bever, T. G., \& Poeppel, D. (2010). Analysis by synthesis: a (re-) emerging program of research for language and vision. Biolinguistics, 4(2-3), 174-200.

Brainard, D. H. (1997). The psychophysics toolbox. Spatial vision, 10(4), 433-436.

Brennan, J. R., \& Martin, A. E. (2020). Phase synchronization varies systematically with linguistic structure composition. Philosophical Transactions of the Royal Society B, 375(1791), 20190305.

Brodbeck, C., Hong, L. E., \& Simon, J. Z. (2018). Rapid transformation from auditory to linguistic representations of continuous speech. Current Biology, 28(24), 3976-3983. e3975.

Brown, M., Tanenhaus, M. K., \& Dilley, L. (2021). Syllable inference as a mechanism for spoken language understanding. Topics in Cognitive Science, 13(2), 351-398.

Bruns, A., Eckhorn, R., Jokeit, H., \& Ebner, A. (2000). Amplitude envelope correlation detects coupling among incoherent brain signals. Neuroreport, 11(7), 1509-1514.

Cabral, J., Kringelbach, M. L., \& Deco, G. (2014). Exploring the network dynamics underlying brain activity during rest. Progress in neurobiology, 114, 102-131.

Canolty, R. T., Edwards, E., Dalal, S. S., Soltani, M., Nagarajan, S. S., Kirsch, H. E., . . Knight, R. T. (2006). High gamma power is phase-locked to theta oscillations in human neocortex. Science, 313(5793), 1626-1628.

Catford, J. C. (1988). A practical introduction to phonetics: Clarendon Press Oxford.

Chang, C.-Y., Hsu, S.-H., Pion-Tonachini, L., \& Jung, T.-P. (2018). Evaluation of artifact subspace reconstruction for automatic EEG artifact removal. Paper presented at the 2018 40th Annual International Conference of the IEEE Engineering in Medicine and Biology Society (EMBC).

Cohen, M. X. (2011). Error-related medial frontal theta activity predicts cingulate-related structural connectivity. Neuroimage, 55(3), 1373-1383.

Cohen, M. X. (2014). Analyzing neural time series data: theory and practice: MIT press.

Cohen, M. X. (2015). Effects of time lag and frequency matching on phase-based connectivity. Journal of neuroscience methods, 250, 137-146.

Coulson, S., King, J. W., \& Kutas, M. (1998). Expect the unexpected: Event-related brain response to morphosyntactic violations. Language and Cognitive Processes, 13(1), 21-58.

Crosse, M. J., Di Liberto, G. M., Bednar, A., \& Lalor, E. C. (2016). The multivariate temporal response function (mTRF) toolbox: a MATLAB toolbox for relating neural signals to continuous stimuli. Frontiers in human neuroscience, 10, 604.

Crosse, M. J., \& Lalor, E. C. (2014). The cortical representation of the speech envelope is earlier for audiovisual speech than audio speech. Journal of neurophysiology, 111(7), 1400-1408.

de Bode, S., Smets, L., Mathern, G. W., \& Dubinsky, S. (2015). Complex syntax in the isolated right hemisphere: Receptive grammatical abilities after cerebral hemispherectomy. Epilepsy \& Behavior, 51, 33-39. 
Delorme, A., \& Makeig, S. (2004). EEGLAB: an open source toolbox for analysis of single-trial EEG dynamics including independent component analysis. Journal of neuroscience methods, 134(1), 9-21.

Di Liberto, G. M., \& Lalor, E. C. (2017). Indexing cortical entrainment to natural speech at the phonemic level: Methodological considerations for applied research. Hearing research, 348, 70-77.

Di Liberto, G. M., O'Sullivan, J. A., \& Lalor, E. C. (2015). Low-frequency cortical entrainment to speech reflects phoneme-level processing. Current Biology, 25(19), 2457-2465.

Dimitrijevic, A., Smith, M. L., Kadis, D. S., \& Moore, D. R. (2017). Cortical alpha oscillations predict speech intelligibility. Frontiers in human neuroscience, 11, 88.

Ding, N., Melloni, L., Yang, A., Wang, Y., Zhang, W., \& Poeppel, D. (2017). Characterizing neural entrainment to hierarchical linguistic units using electroencephalography (EEG). Frontiers in human neuroscience, $11,481$.

Ding, N., Melloni, L., Zhang, H., Tian, X., \& Poeppel, D. (2016). Cortical tracking of hierarchical linguistic structures in connected speech. Nature neuroscience, 19(1), 158-164.

Ding, N., Patel, A. D., Chen, L., Butler, H., Luo, C., \& Poeppel, D. (2017). Temporal modulations in speech and music. Neuroscience \& Biobehavioral Reviews, 81, 181-187.

Ding, N., \& Simon, J. Z. (2012a). Emergence of neural encoding of auditory objects while listening to competing speakers. Proceedings of the National Academy of Sciences, 109(29), 11854-11859.

Ding, N., \& Simon, J. Z. (2012b). Neural coding of continuous speech in auditory cortex during monaural and dichotic listening. Journal of neurophysiology, 107(1), 78-89.

Ding, N., \& Simon, J. Z. (2013). Adaptive temporal encoding leads to a background-insensitive cortical representation of speech. Journal of Neuroscience, 33(13), 5728-5735.

Doelling, K. B., Arnal, L. H., Ghitza, O., \& Poeppel, D. (2014). Acoustic landmarks drive delta-theta oscillations to enable speech comprehension by facilitating perceptual parsing. Neuroimage, 85 , 761-768.

Donhauser, P. W., \& Baillet, S. (2020). Two distinct neural timescales for predictive speech processing. Neuron, 105(2), 385-393. e389.

Doumas, L. A., \& Martin, A. E. (2018). Learning structured representations from experience Psychology of Learning and Motivation (Vol. 69, pp. 165-203): Elsevier.

Friederici, A. D. (1995). The time course of syntactic activation during language processing: A model based on neuropsychological and neurophysiological data. Brain and language, 50(3), 259-281.

Friederici, A. D., Pfeifer, E., \& Hahne, A. (1993). Event-related brain potentials during natural speech processing: Effects of semantic, morphological and syntactic violations. Cognitive brain research, 1(3), 183-192.

Ghitza, O., Giraud, A.-L., \& Poeppel, D. (2013). Neuronal oscillations and speech perception: critical-band temporal envelopes are the essence. Frontiers in human neuroscience, 6, 340.

Giraud, A.-L., Kleinschmidt, A., Poeppel, D., Lund, T. E., Frackowiak, R. S., \& Laufs, H. (2007). Endogenous cortical rhythms determine cerebral specialization for speech perception and production. Neuron, 56(6), 1127-1134.

Giraud, A.-L., \& Poeppel, D. (2012). Cortical oscillations and speech processing: emerging computational principles and operations. Nature neuroscience, 15(4), 511.

Golumbic, E. M. Z., Ding, N., Bickel, S., Lakatos, P., Schevon, C. A., McKhann, G. M., . . Simon, J. Z. (2013). Mechanisms underlying selective neuronal tracking of attended speech at a "cocktail party". Neuron, 77(5), 980-991.

Greenwood, D. D. (1990). A cochlear frequency-position function for several species-29 years later. The Journal of the Acoustical Society of America, 87(6), 2592-2605.

Grodzinsky, Y. (2000). The neurology of syntax: Language use without Broca's area. Behavioral and brain sciences, 23(1), 1-21. 
Grodzinsky, Y., \& Friederici, A. D. (2006). Neuroimaging of syntax and syntactic processing. Current opinion in neurobiology, 16(2), 240-246.

Gui, P., Jiang, Y., Zang, D., Qi, Z., Tan, J., Tanigawa, H., . . Zhao, J. (2020). Assessing the depth of language processing in patients with disorders of consciousness. Nature neuroscience, 1-10.

Gwilliams, L., \& King, J.-R. (2020). Recurrent processes support a cascade of hierarchical decisions. Elife, 9, e56603.

Haegens, S., Osipova, D., Oostenveld, R., \& Jensen, O. (2010). Somatosensory working memory performance in humans depends on both engagement and disengagement of regions in a distributed network. Human brain mapping, 31(1), 26-35.

Hagoort, P. (2013). MUC (memory, unification, control) and beyond. Frontiers in psychology, 4, 416.

Hagoort, P., Brown, C., \& Groothusen, J. (1993). The syntactic positive shift (SPS) as an ERP measure of syntactic processing. Language and Cognitive Processes, 8(4), 439-483.

Halle, M., \& Stevens, K. (1962). Speech recognition: A model and a program for research. IRE transactions on information theory, 8(2), 155-159.

Halle, M., Stevens, K. N., Wathen-Dunn, W., \& Woods, L. (1959). Proceedings of the Seminar on Speech Compression and Processing.

Hipp, J. F., Hawellek, D. J., Corbetta, M., Siegel, M., \& Engel, A. K. (2012). Large-scale cortical correlation structure of spontaneous oscillatory activity. Nature neuroscience, 15(6), 884-890.

Howard, M. F., \& Poeppel, D. (2010). Discrimination of speech stimuli based on neuronal response phase patterns depends on acoustics but not comprehension. Journal of neurophysiology, 104(5), 2500-2511.

Hutchison, R. M., Womelsdorf, T., Allen, E. A., Bandettini, P. A., Calhoun, V. D., Corbetta, M., . . . Gonzalez-Castillo, J. (2013). Dynamic functional connectivity: promise, issues, and interpretations. Neuroimage, 80, 360-378.

Jeans, J. H. (1968). Science \& music: Courier Corporation.

Jin, P., Lu, Y., \& Ding, N. (2020). Low-frequency neural activity reflects rule-based chunking during speech listening. Elife, 9, e55613.

Jin, P., Zou, J., Zhou, T., \& Ding, N. (2018). Eye activity tracks task-relevant structures during speech and auditory sequence perception. Nature communications, 9(1), 1-15.

Kaufeld, G., Bosker, H. R., Ten Oever, S., Alday, P. M., Meyer, A. S., \& Martin, A. E. (2020). Linguistic structure and meaning organize neural oscillations into a content-specific hierarchy. Journal of Neuroscience, 40(49), 9467-9475.

Keitel, A., Gross, J., \& Kayser, C. (2018). Perceptually relevant speech tracking in auditory and motor cortex reflects distinct linguistic features. PLoS biology, 16(3), e2004473.

Kerlin, J. R., Shahin, A. J., \& Miller, L. M. (2010). Attentional gain control of ongoing cortical speech representations in a "cocktail party". Journal of Neuroscience, 30(2), 620-628.

Khalighinejad, B., da Silva, G. C., \& Mesgarani, N. (2017). Dynamic encoding of acoustic features in neural responses to continuous speech. Journal of Neuroscience, 37(8), 2176-2185.

Klimesch, W., Sauseng, P., \& Hanslmayr, S. (2007). EEG alpha oscillations: the inhibition-timing hypothesis. Brain research reviews, 53(1), 63-88.

Kösem, A., \& Van Wassenhove, V. (2017). Distinct contributions of low-and high-frequency neural oscillations to speech comprehension. Language, Cognition and Neuroscience, 32(5), 536-544.

Kothe, C. A. E., \& Jung, T.-p. (2016). Artifact removal techniques with signal reconstruction: Google Patents.

Lachaux, J.-P., Rodriguez, E., Le Van Quyen, M., Lutz, A., Martinerie, J., \& Varela, F. J. (2000). Studying single-trials of phase synchronous activity in the brain. International Journal of Bifurcation and Chaos, 10(10), 2429-2439. 
Lachaux, J. P., Rodriguez, E., Martinerie, J., \& Varela, F. J. (1999). Measuring phase synchrony in brain signals. Human brain mapping, 8(4), 194-208.

Luo, H., \& Poeppel, D. (2007). Phase patterns of neuronal responses reliably discriminate speech in human auditory cortex. Neuron, 54(6), 1001-1010.

Maess, B., Koelsch, S., Gunter, T. C., \& Friederici, A. D. (2001). Musical syntax is processed in Broca's area: an MEG study. Nature neuroscience, 4(5), 540-545.

Maris, E., \& Oostenveld, R. (2007). Nonparametric statistical testing of EEG-and MEG-data. Journal of neuroscience methods, 164(1), 177-190.

Marslen-Wilson, W., \& Tyler, L. K. (1980). The temporal structure of spoken language understanding. Cognition, 8(1), 1-71.

Marslen-Wilson, W. D. (1987). Functional parallelism in spoken word-recognition. Cognition, 25(1-2), 71102.

Marslen-Wilson, W. D., \& Welsh, A. (1978). Processing interactions and lexical access during word recognition in continuous speech. Cognitive psychology, 10(1), 29-63.

Martin, A. E. (2016). Language processing as cue integration: Grounding the psychology of language in perception and neurophysiology. Frontiers in psychology, 7, 120.

Martin, A. E. (2020). A compositional neural architecture for language. Journal of Cognitive Neuroscience, 32(8), 1407-1427.

Martin, A. E., \& Doumas, L. A. (2017). A mechanism for the cortical computation of hierarchical linguistic structure. PLoS biology, 15(3), e2000663.

Martin, A. E., \& Doumas, L. A. (2019). Predicate learning in neural systems: using oscillations to discover latent structure. Current Opinion in Behavioral Sciences, 29, 77-83.

Martin, A. E., \& Doumas, L. A. (2020). Tensors and compositionality in neural systems. Philosophical Transactions of the Royal Society B, 375(1791), 20190306.

Meyer, L. (2018). The neural oscillations of speech processing and language comprehension: state of the art and emerging mechanisms. European Journal of Neuroscience, 48(7), 2609-2621.

Meyer, L., \& Gumbert, M. (2018). Synchronization of electrophysiological responses with speech benefits syntactic information processing. Journal of Cognitive Neuroscience, 30(8), 1066-1074.

Meyer, L., Henry, M. J., Gaston, P., Schmuck, N., \& Friederici, A. D. (2017). Linguistic bias modulates interpretation of speech via neural delta-band oscillations. Cerebral Cortex, 27(9), 4293-4302.

Meyer, L., Sun, Y., \& Martin, A. E. (2020). Synchronous, but not entrained: exogenous and endogenous cortical rhythms of speech and language processing. Language, Cognition and Neuroscience, 35(9), 1089-1099.

Morillon, B., Liégeois-Chauvel, C., Arnal, L. H., Bénar, C. G., \& Giraud, A.-L. (2012). Asymmetric function of theta and gamma activity in syllable processing: an intra-cortical study. Frontiers in psychology, 3, 248.

Mormann, F., Lehnertz, K., David, P., \& Elger, C. E. (2000). Mean phase coherence as a measure for phase synchronization and its application to the EEG of epilepsy patients. Physica D: Nonlinear Phenomena, 144(3-4), 358-369.

Neuper, C., Wörtz, M., \& Pfurtscheller, G. (2006). ERD/ERS patterns reflecting sensorimotor activation and deactivation. Progress in brain research, 159, 211-222.

Obleser, J., \& Kayser, C. (2019). Neural entrainment and attentional selection in the listening brain. Trends in cognitive sciences, 23(11), 913-926.

Obleser, J., \& Weisz, N. (2012). Suppressed alpha oscillations predict intelligibility of speech and its acoustic details. Cerebral Cortex, 22(11), 2466-2477.

Obleser, J., Wöstmann, M., Hellbernd, N., Wilsch, A., \& Maess, B. (2012). Adverse listening conditions and memory load drive a common alpha oscillatory network. Journal of Neuroscience, 32(36), 12376-12383. 
Oostenveld, R., Fries, P., Maris, E., \& Schoffelen, J.-M. (2011). FieldTrip: open source software for advanced analysis of MEG, EEG, and invasive electrophysiological data. Computational intelligence and neuroscience, 2011.

Osterhout, L., \& Holcomb, P. J. (1992). Event-related brain potentials elicited by syntactic anomaly. Journal of memory and language, 31(6), 785-806.

Osterhout, L., \& Mobley, L. A. (1995). Event-related brain potentials elicited by failure to agree. Journal of memory and language, 34(6), 739-773.

Patel, A. D., Gibson, E., Ratner, J., Besson, M., \& Holcomb, P. J. (1998). Processing syntactic relations in language and music: An event-related potential study. Journal of Cognitive Neuroscience, 10(6), 717-733.

Peelle, J. E., \& Davis, M. H. (2012). Neural oscillations carry speech rhythm through to comprehension. Frontiers in psychology, 3, 320.

Peelle, J. E., Gross, J., \& Davis, M. H. (2013). Phase-locked responses to speech in human auditory cortex are enhanced during comprehension. Cerebral Cortex, 23(6), 1378-1387.

Pellegrino, F., Coupé, C., \& Marsico, E. (2011). A cross-language perspective on speech information rate. Language, 539-558.

Peña, M., \& Melloni, L. (2012). Brain oscillations during spoken sentence processing. Journal of Cognitive Neuroscience, 24(5), 1149-1164.

Perrin, F., Bertrand, O., \& Pernier, J. (1987). Scalp current density mapping: value and estimation from potential data. IEEE Transactions on Biomedical Engineering(4), 283-288.

Perrin, F., Pernier, J., Bertnard, O., Giard, M.-H., \& Echallier, J. (1987). Mapping of scalp potentials by surface spline interpolation. Electroencephalography and clinical neurophysiology, 66(1), 75-81.

Pfurtscheller, G., Brunner, C., Schlögl, A., \& Da Silva, F. L. (2006). Mu rhythm (de) synchronization and EEG single-trial classification of different motor imagery tasks. Neuroimage, 31(1), 153-159.

Pfurtscheller, G., Neuper, C., Schlogl, A., \& Lugger, K. (1998). Separability of EEG signals recorded during right and left motor imagery using adaptive autoregressive parameters. IEEE transactions on Rehabilitation Engineering, 6(3), 316-325.

Phillips, C. (2003). Linear order and constituency. Linguistic inquiry, 34(1), 37-90.

Poeppel, D. (2003). The analysis of speech in different temporal integration windows: cerebral lateralization as 'asymmetric sampling in time'. Speech communication, 41(1), 245-255.

Poeppel, D., \& Assaneo, M. F. (2020). Speech rhythms and their neural foundations. Nature reviews neuroscience, 21(6), 322-334.

Poeppel, D., \& Monahan, P. J. (2011). Feedforward and feedback in speech perception: Revisiting analysis by synthesis. Language and Cognitive Processes, 26(7), 935-951.

Puvvada, K. C., \& Simon, J. Z. (2017). Cortical representations of speech in a multitalker auditory scene. Journal of Neuroscience, 37(38), 9189-9196.

Rimmele, J. M., Morillon, B., Poeppel, D., \& Arnal, L. H. (2018). Proactive sensing of periodic and aperiodic auditory patterns. Trends in cognitive sciences, 22(10), 870-882.

Schlögl, A., Lee, F., Bischof, H., \& Pfurtscheller, G. (2005). Characterization of four-class motor imagery $\mathrm{EEG}$ data for the $\mathrm{BCl}$-competition 2005. Journal of neural engineering, 2(4), L14.

Shannon, R. V., Zeng, F.-G., Kamath, V., Wygonski, J., \& Ekelid, M. (1995). Speech recognition with primarily temporal cues. Science, 270(5234), 303-304.

Smith, Z. M., Delgutte, B., \& Oxenham, A. J. (2002). Chimaeric sounds reveal dichotomies in auditory perception. Nature, 416(6876), 87-90.

Snodgrass, J. G., \& Vanderwart, M. (1980). A standardized set of 260 pictures: norms for name agreement, image agreement, familiarity, and visual complexity. Journal of experimental psychology: Human learning and memory, 6(2), 174.

Sporns, O. (2010). Networks of the Brain: MIT press. 
Stam, C. J., Nolte, G., \& Daffertshofer, A. (2007). Phase lag index: assessment of functional connectivity from multi channel EEG and MEG with diminished bias from common sources. Human brain mapping, 28(11), 1178-1193.

Strauß, A., Wöstmann, M., \& Obleser, J. (2014). Cortical alpha oscillations as a tool for auditory selective inhibition. Frontiers in human neuroscience, 8, 350.

Suffczynski, P., Kalitzin, S., Pfurtscheller, G., \& Da Silva, F. L. (2001). Computational model of thalamocortical networks: dynamical control of alpha rhythms in relation to focal attention. International Journal of Psychophysiology, 43(1), 25-40.

Ten Oever, S., De Weerd, P., \& Sack, A. T. (2020). Phase-dependent amplification of working memory content and performance. Nature communications, 11(1), 1-8.

Tikhonov, A. N., \& Arsenin, V. Y. (1977). Solutions of ill-posed problems. New York, 1, 30.

Titze, I. R., Baken, R. J., Bozeman, K. W., Granqvist, S., Henrich, N., Herbst, C. T., . . Kent, R. D. (2015). Toward a consensus on symbolic notation of harmonics, resonances, and formants in vocalization. The Journal of the Acoustical Society of America, 137(5), 3005-3007.

Titze, I. R., \& Martin, D. W. (1998). Principles of voice production: Acoustical Society of America.

Varnet, L., Ortiz-Barajas, M. C., Erra, R. G., Gervain, J., \& Lorenzi, C. (2017). A cross-linguistic study of speech modulation spectra. The Journal of the Acoustical Society of America, 142(4), 1976-1989.

Wang, Y., Zhang, J., Zou, J., Luo, H., \& Ding, N. (2019). Prior knowledge guides speech segregation in human auditory cortex. Cerebral Cortex, 29(4), 1561-1571.

Weissbart, H., Kandylaki, K. D., \& Reichenbach, T. (2020). Cortical tracking of surprisal during continuous speech comprehension. Journal of Cognitive Neuroscience, 32(1), 155-166.

Wilsch, A., \& Obleser, J. (2016). What works in auditory working memory? A neural oscillations perspective. Brain research, 1640, 193-207.

Winkler, I., Haufe, S., \& Tangermann, M. (2011). Automatic classification of artifactual ICA-components for artifact removal in EEG signals. Behavioral and Brain Functions, 7(1), 1-15.

Wöstmann, M., Herrmann, B., Maess, B., \& Obleser, J. (2016). Spatiotemporal dynamics of auditory attention synchronize with speech. Proceedings of the National Academy of Sciences, 113(14), 3873-3878.

Wöstmann, M., Herrmann, B., Wilsch, A., \& Obleser, J. (2015). Neural alpha dynamics in younger and older listeners reflect acoustic challenges and predictive benefits. Journal of Neuroscience, 35(4), 1458-1467.

Wöstmann, M., Lim, S.-J., \& Obleser, J. (2017). The human neural alpha response to speech is a proxy of attentional control. Cerebral Cortex, 27(6), 3307-3317.

Zeng, F.-G., Nie, K., Stickney, G. S., Kong, Y.-Y., Vongphoe, M., Bhargave, A., . . Cao, K. (2005). Speech recognition with amplitude and frequency modulations. Proceedings of the National Academy of Sciences, 102(7), 2293-2298.

Zhou, H., Melloni, L., Poeppel, D., \& Ding, N. (2016). Interpretations of frequency domain analyses of neural entrainment: periodicity, fundamental frequency, and harmonics. Frontiers in human neuroscience, 10, 274.

Zoefel, B., Archer-Boyd, A., \& Davis, M. H. (2018). Phase entrainment of brain oscillations causally modulates neural responses to intelligible speech. Current Biology, 28(3), 401-408. e405.

Zoefel, B., \& VanRullen, R. (2016). EEG oscillations entrain their phase to high-level features of speech sound. Neuroimage, 124, 16-23. 

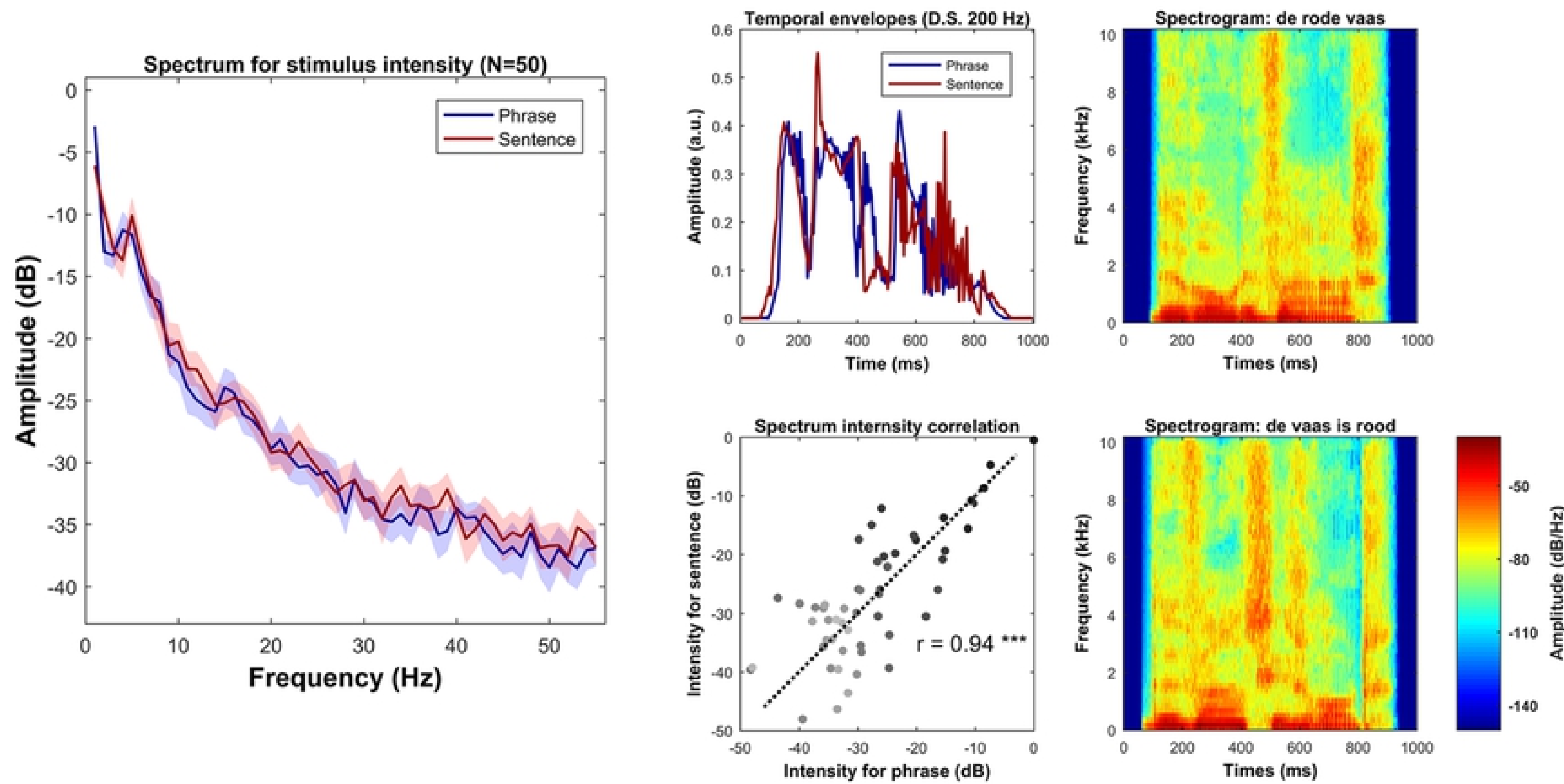

Figure 9 
a

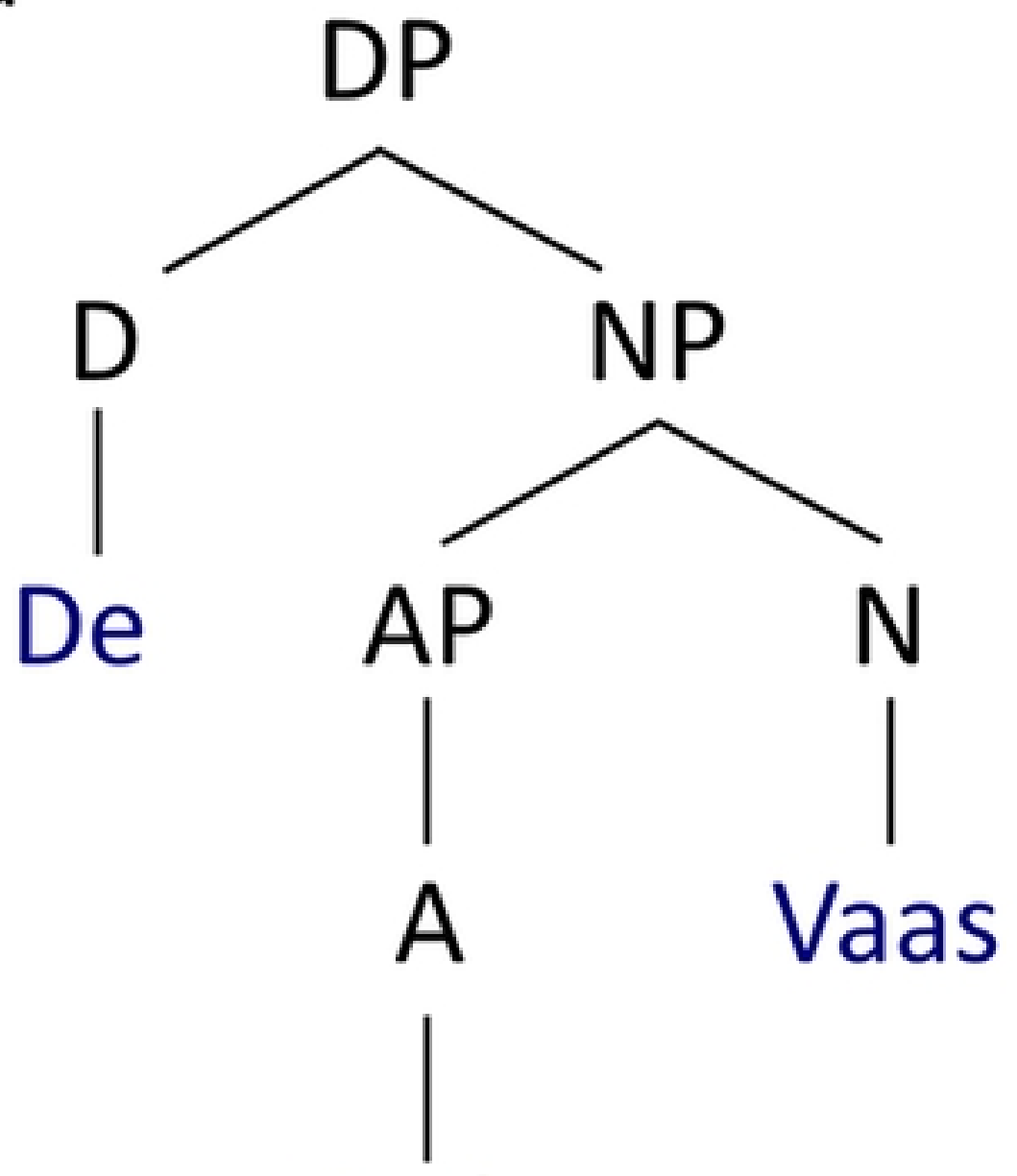

Rode b

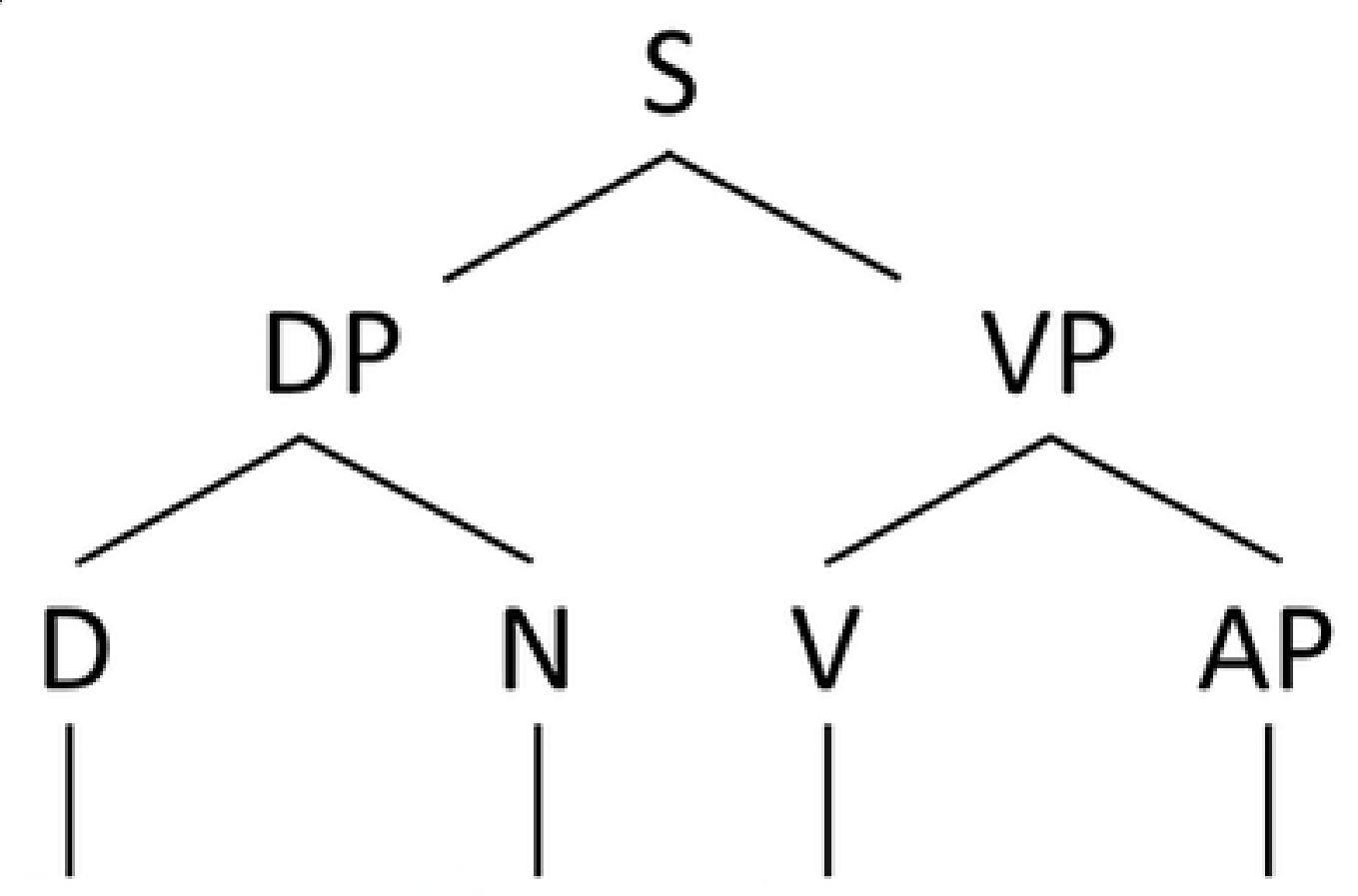

De

Vaas is

Rood

Figure 1 


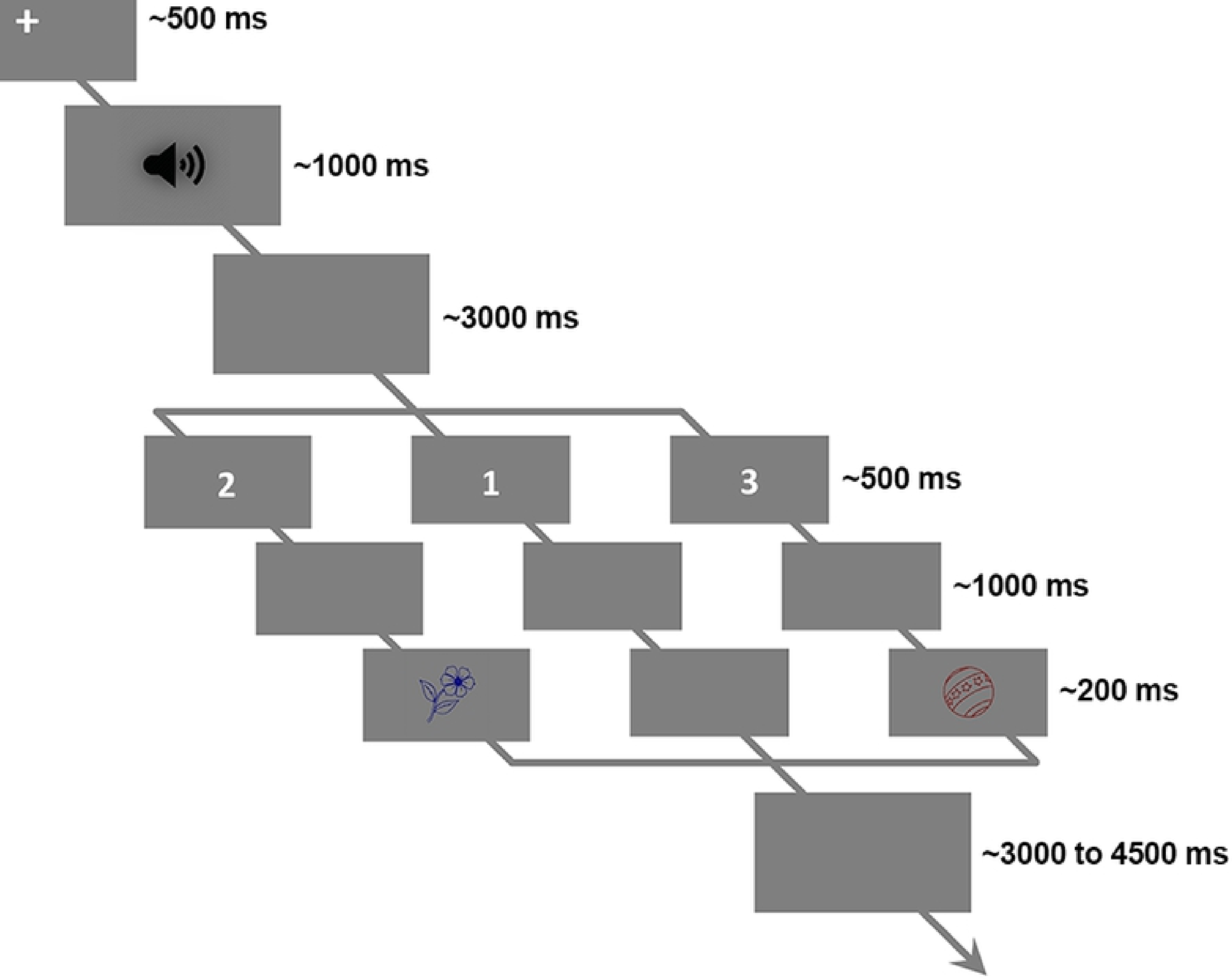

Figure 8 

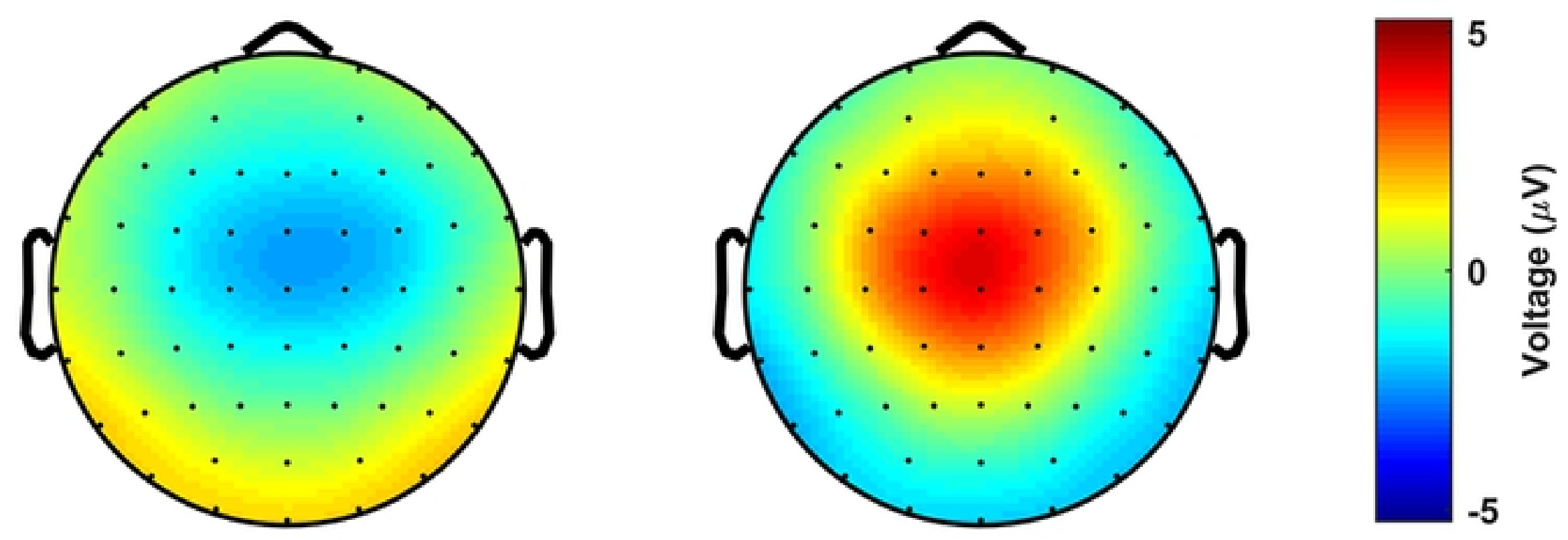

CSD Localizer
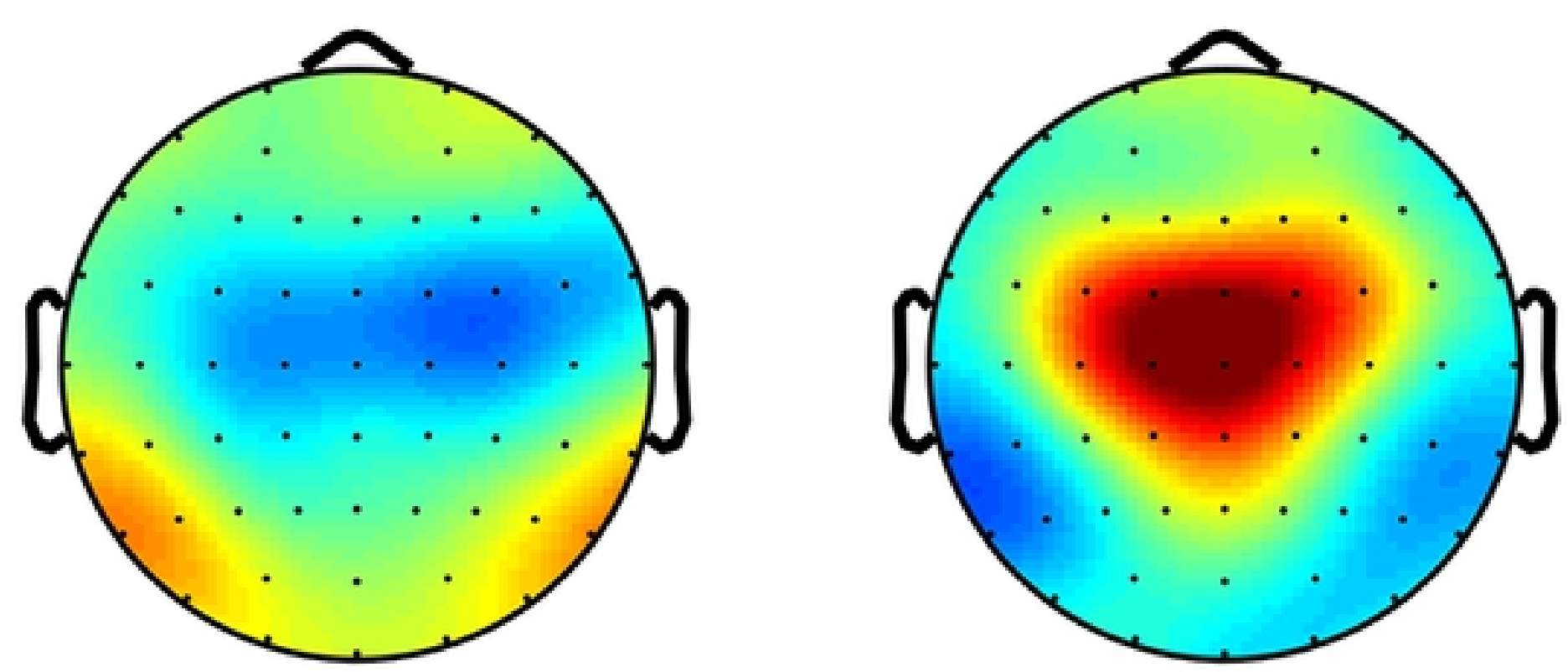

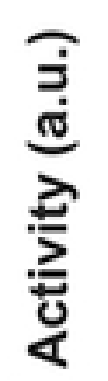

Figure 10 
a
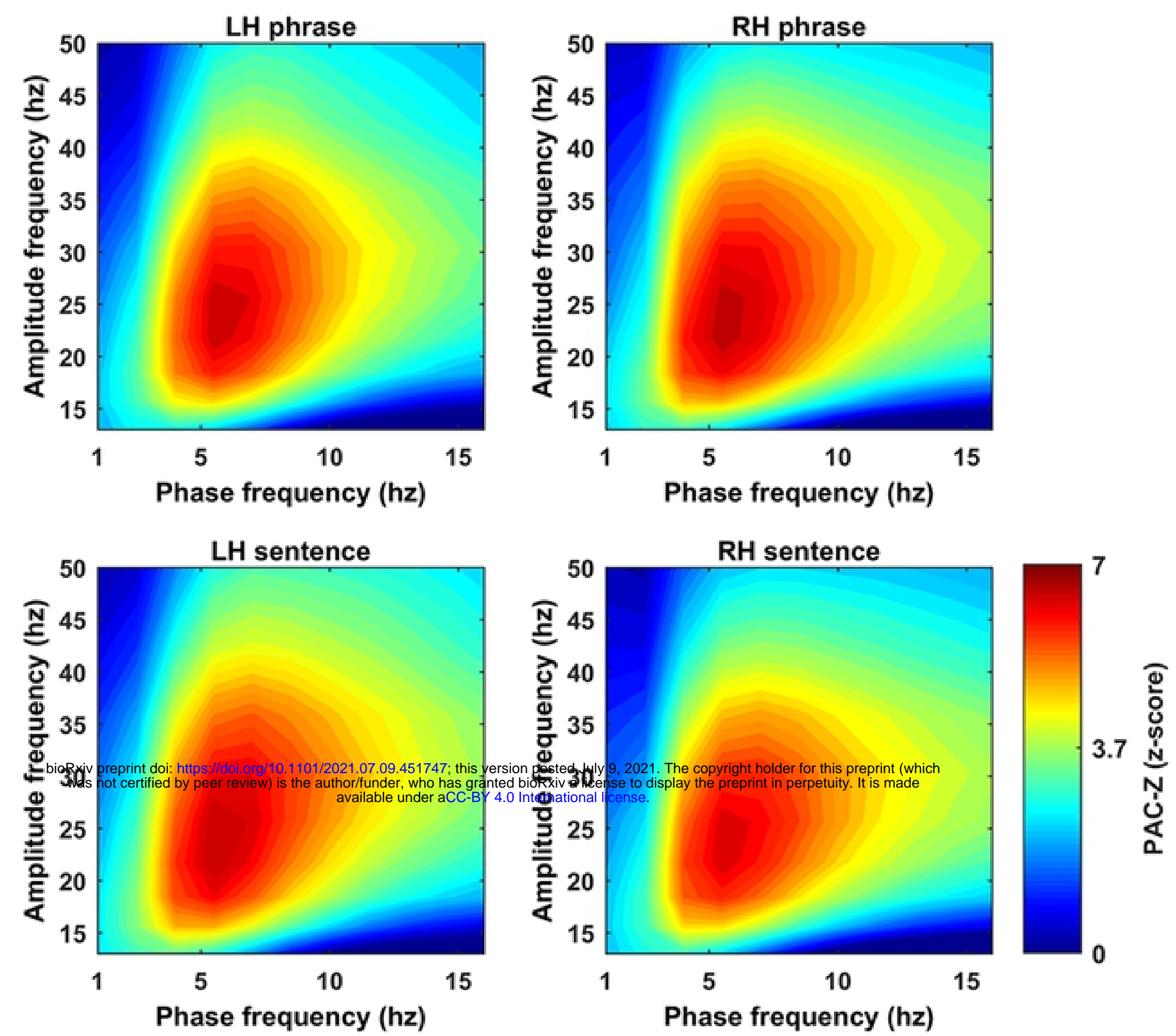

b

Selected sensors

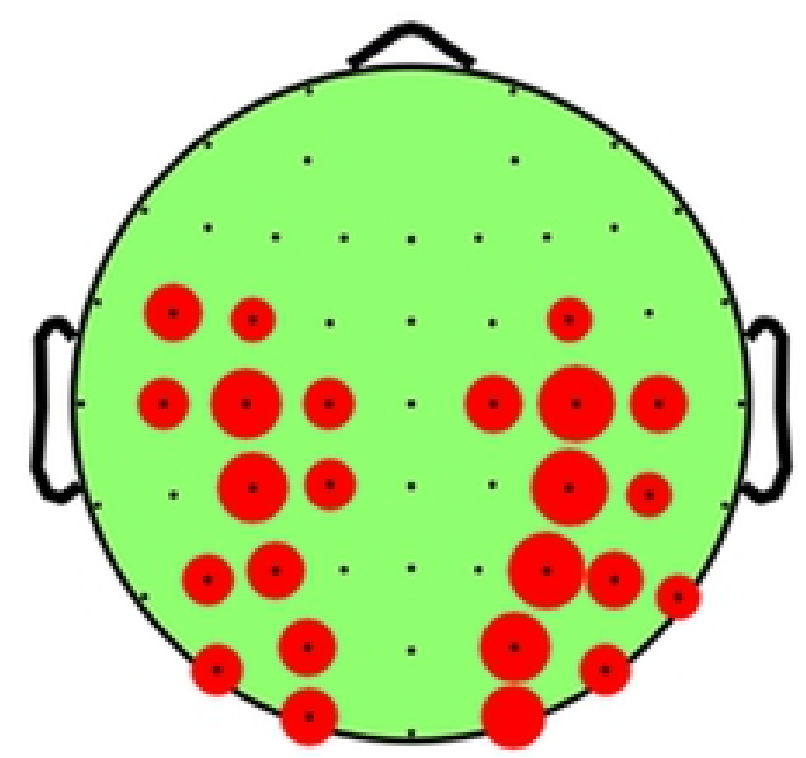

C
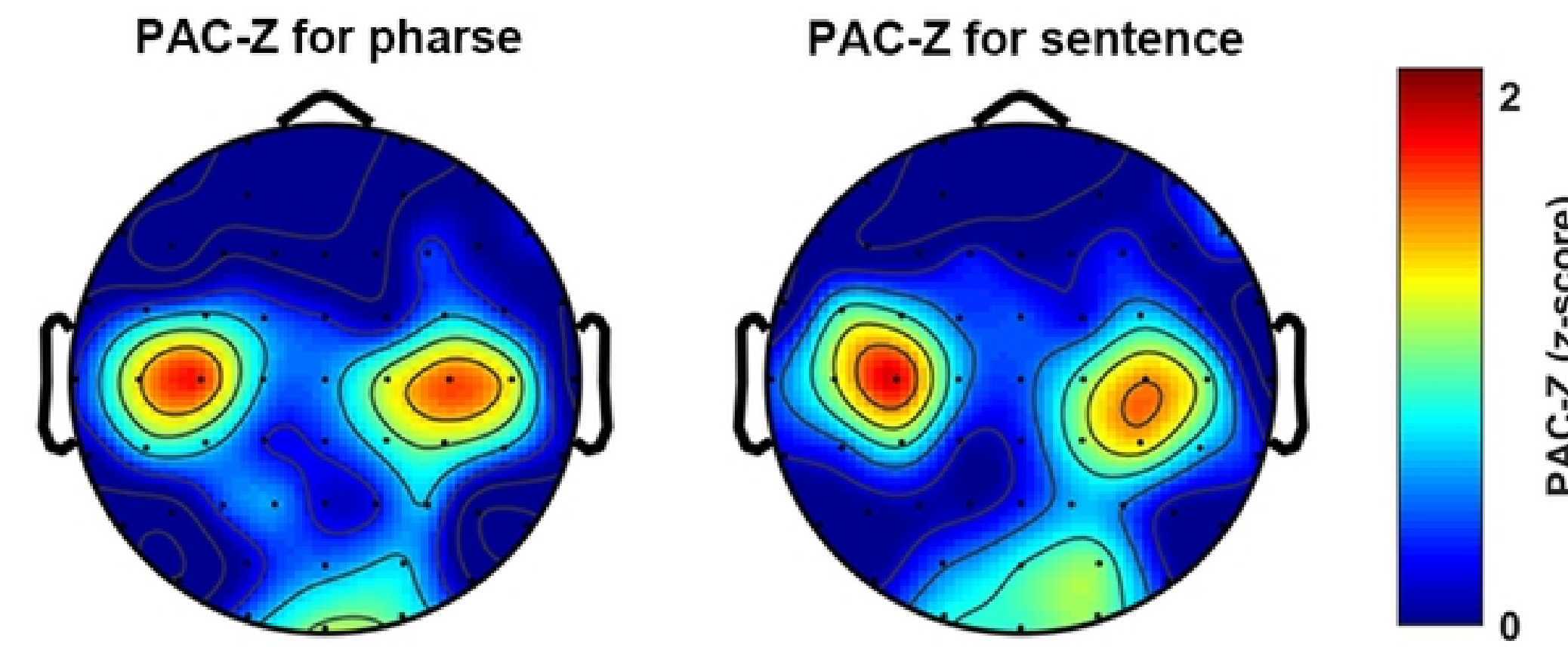

Figure 4 


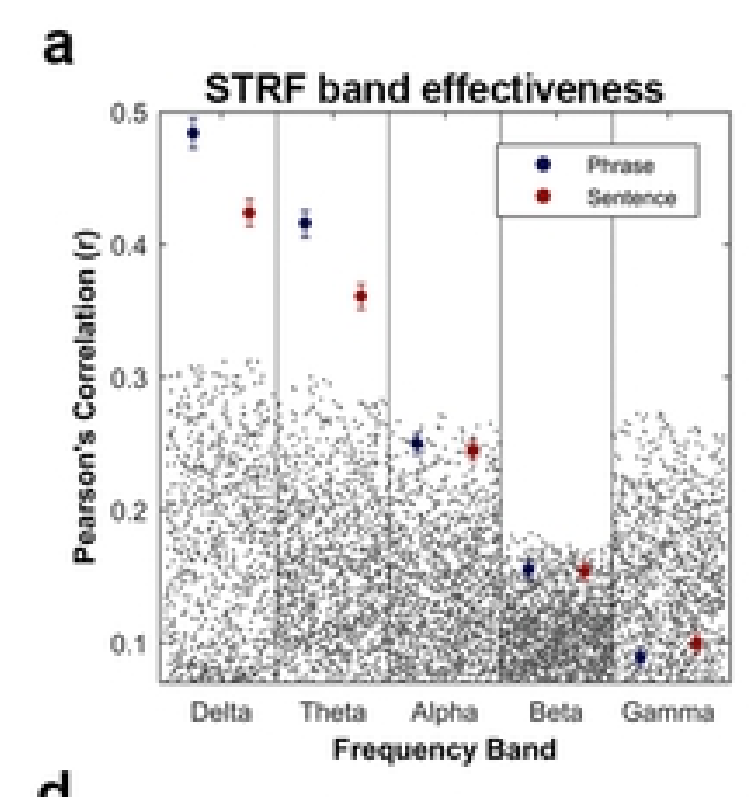

d
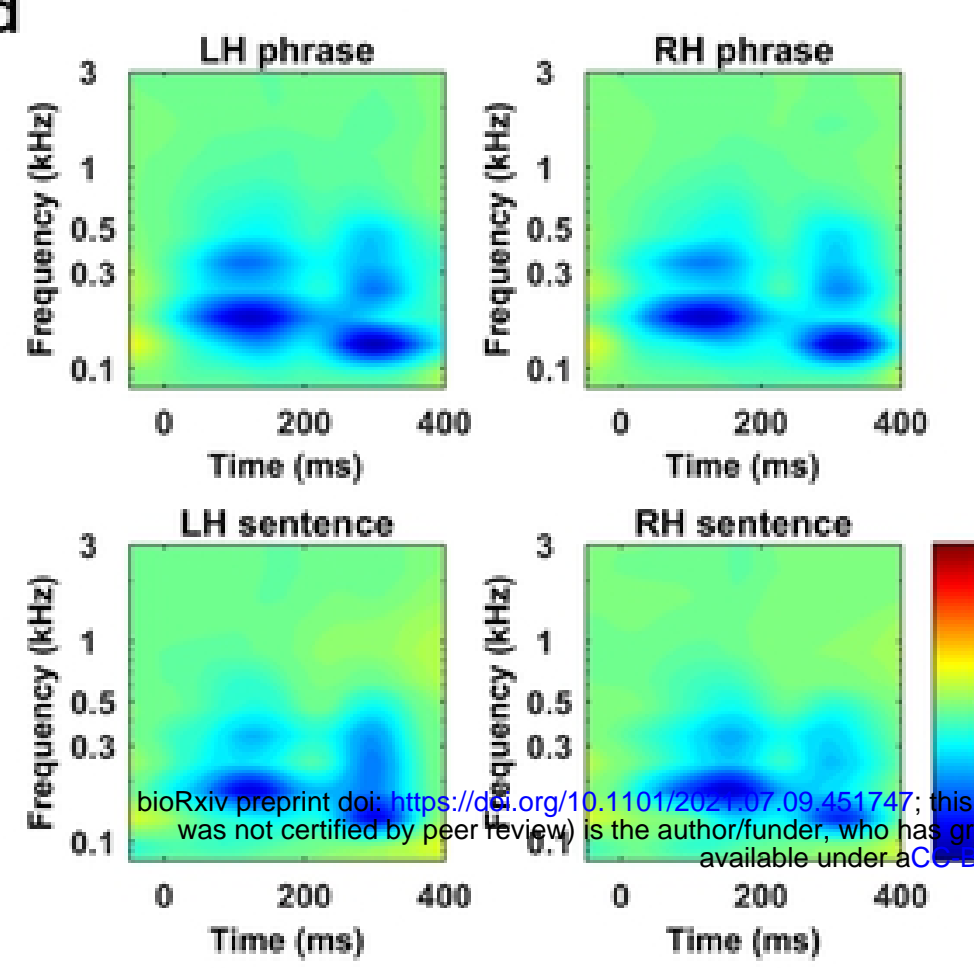

f

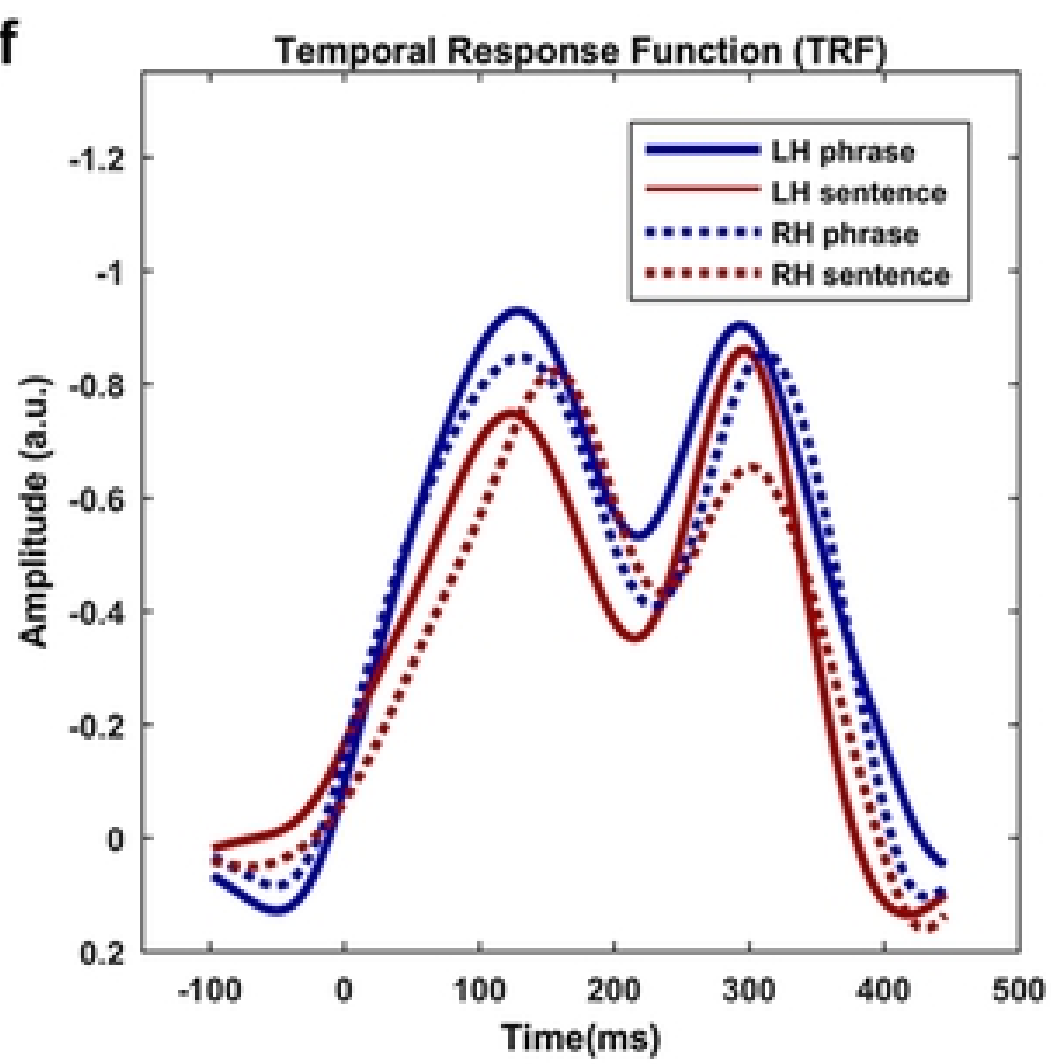

i

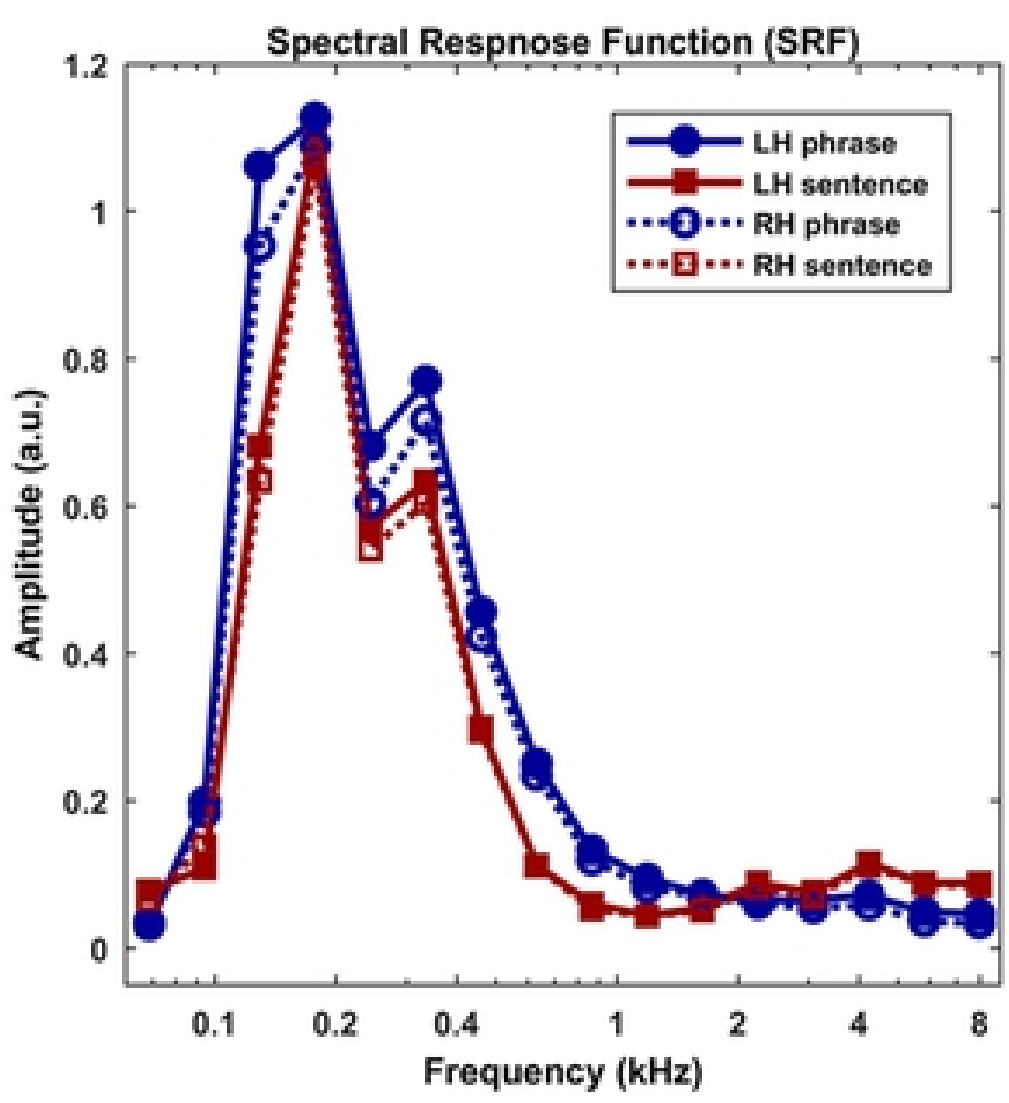

C
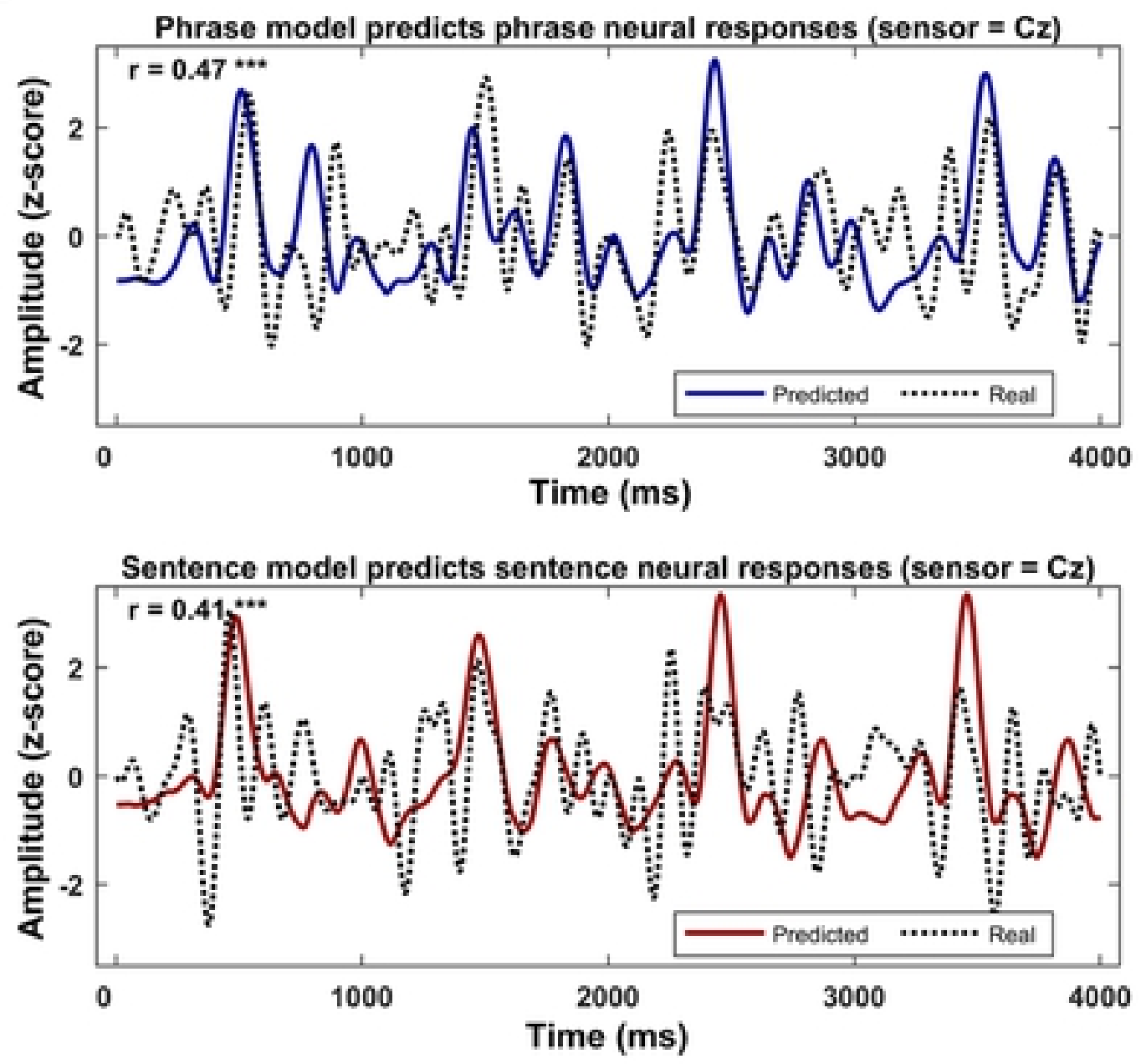

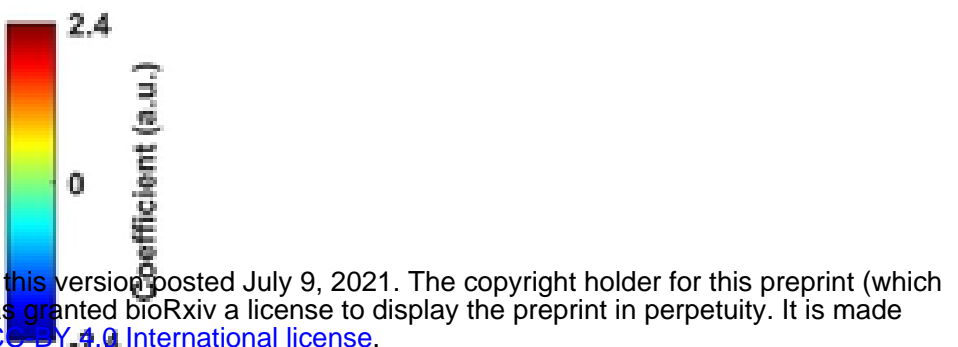
ted bioRxiv a license to display the preprint in perpetuity. It is made

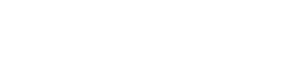

Selected sensors

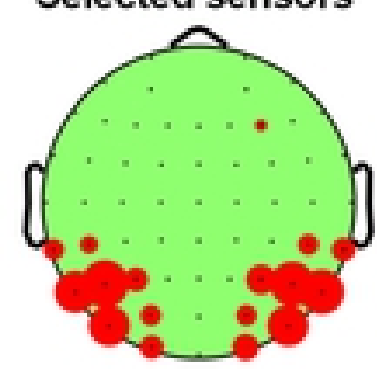

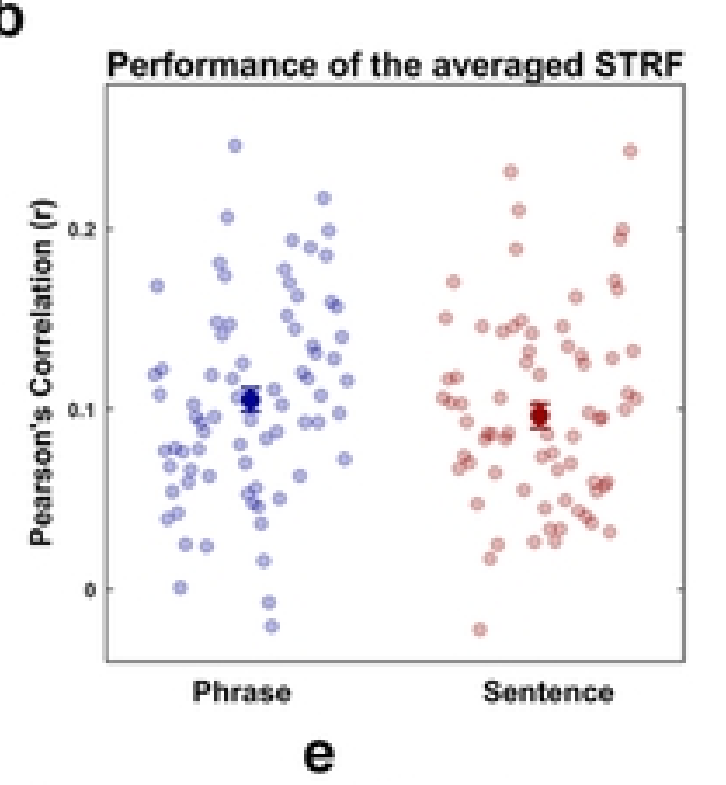

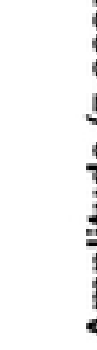


a
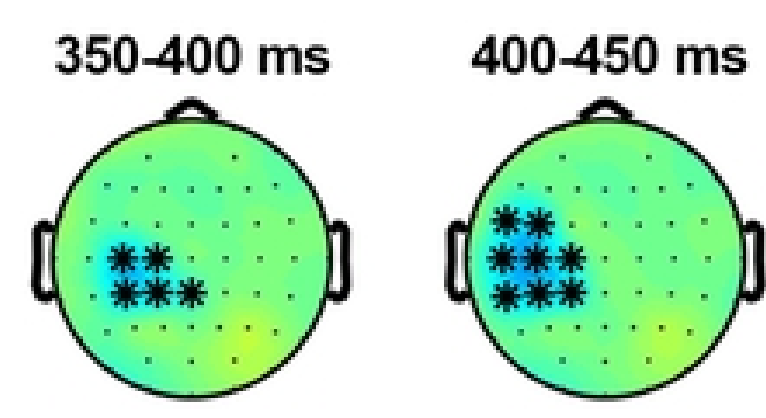

\section{$550-600 \mathrm{~ms}$}
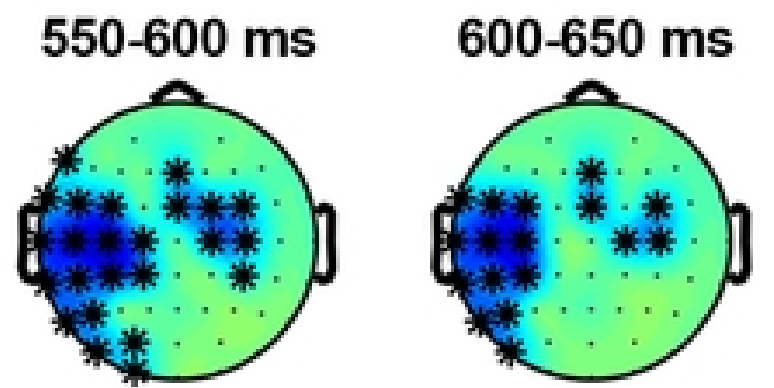

$750-800 \mathrm{~ms}$

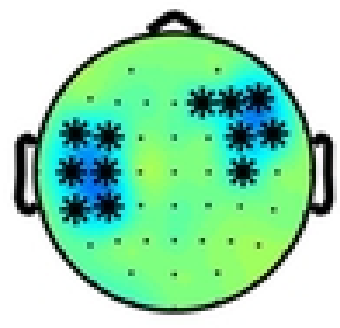

800-850 ms

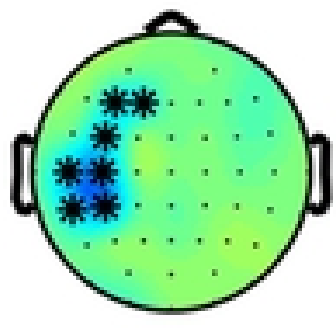

950-1000 ms

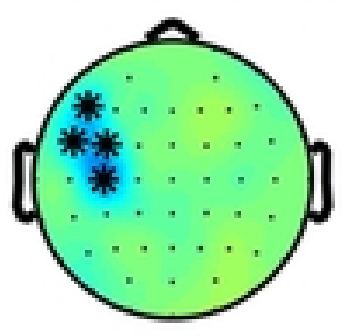

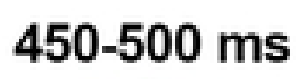

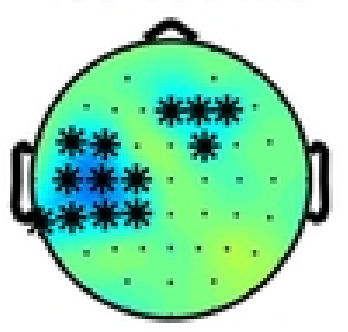

$650-700 \mathrm{~ms}$

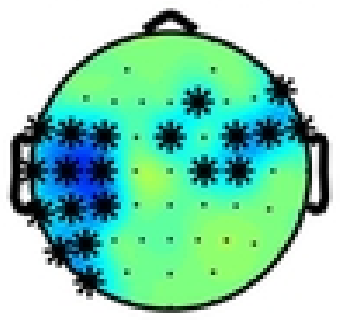

700-750 ms

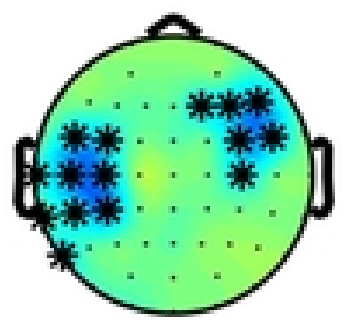

$850-900 \mathrm{~ms}$

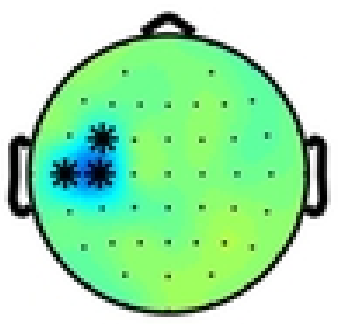

900-950 ms
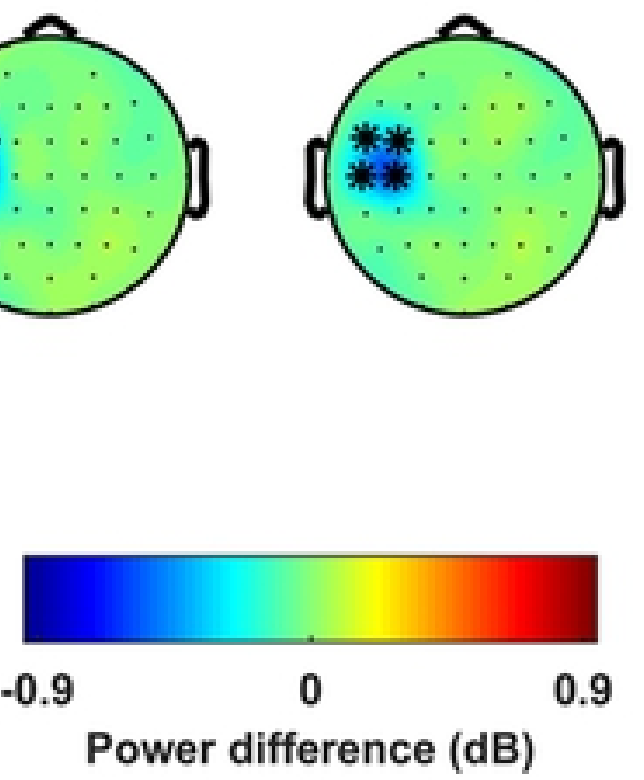

b
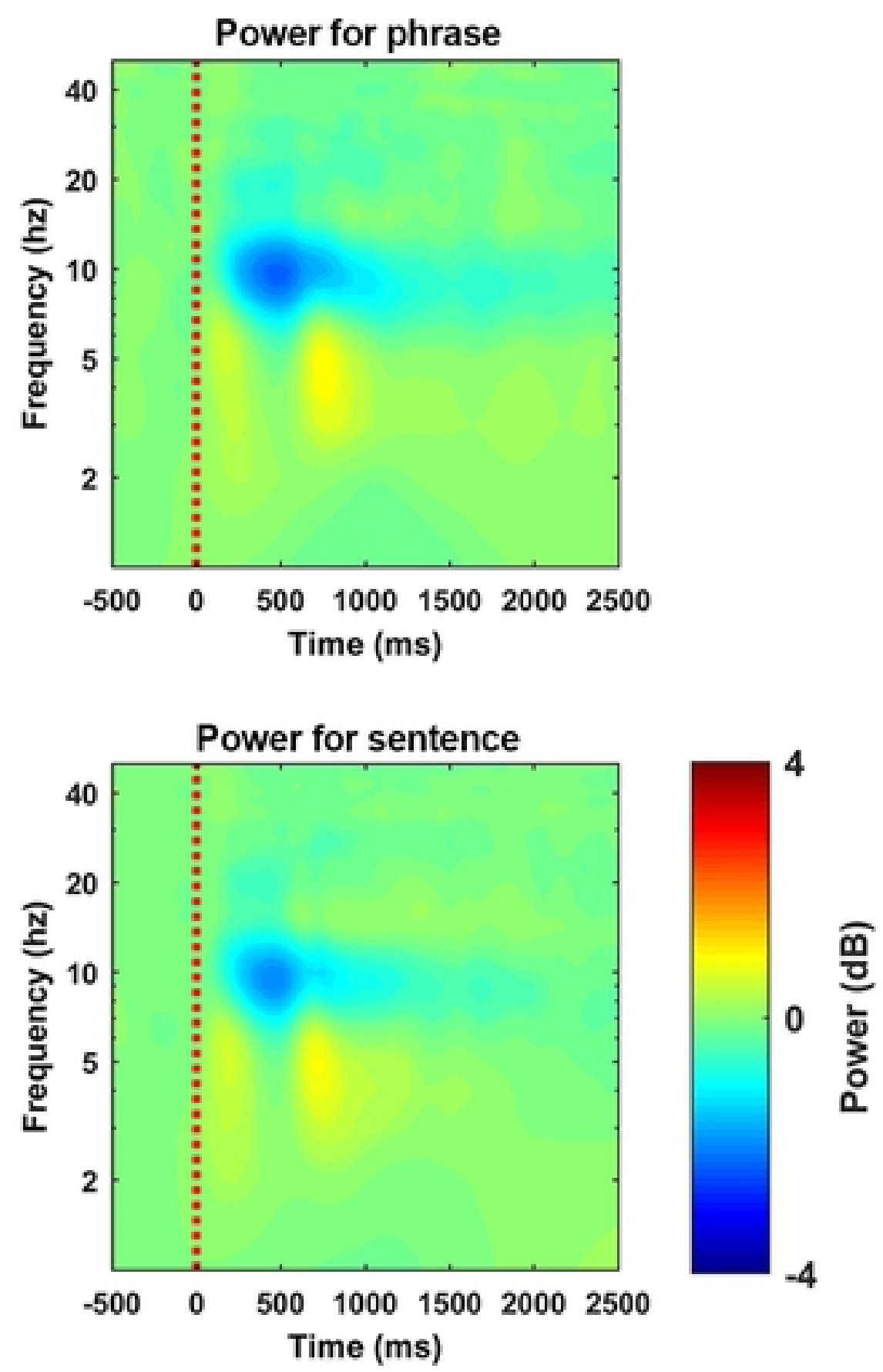

Figure 5 
a
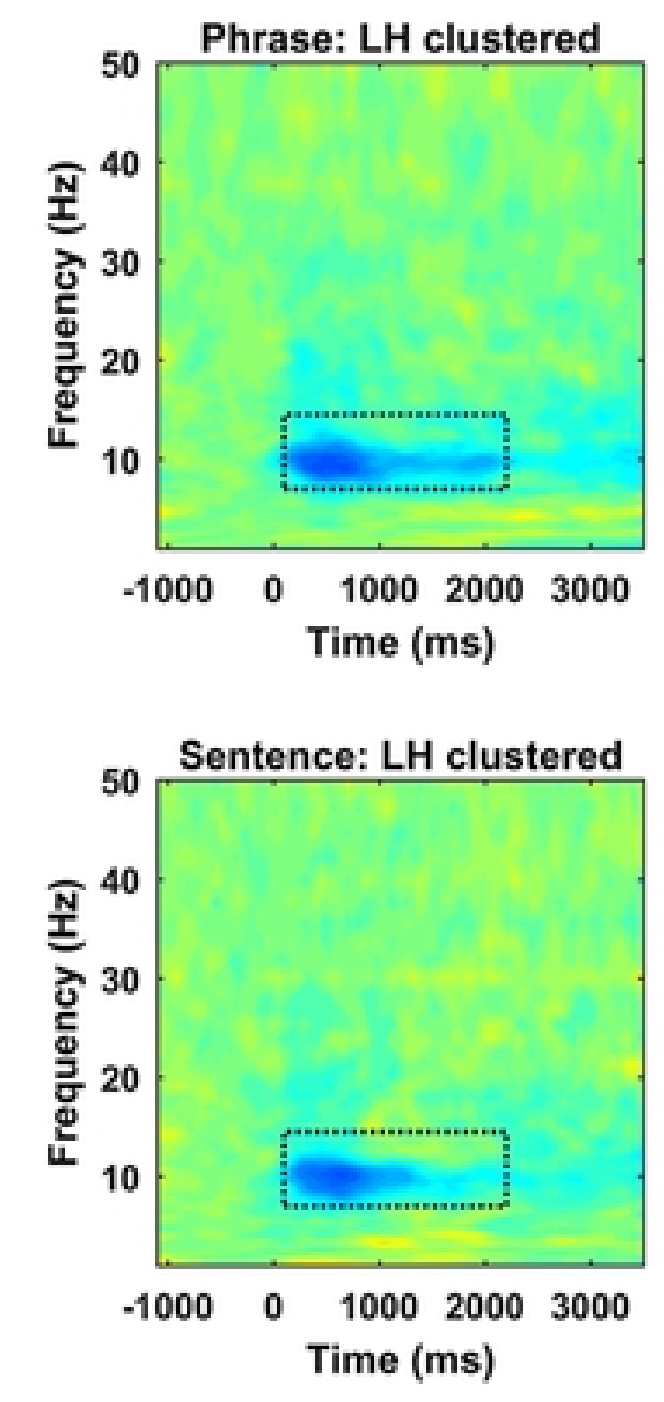
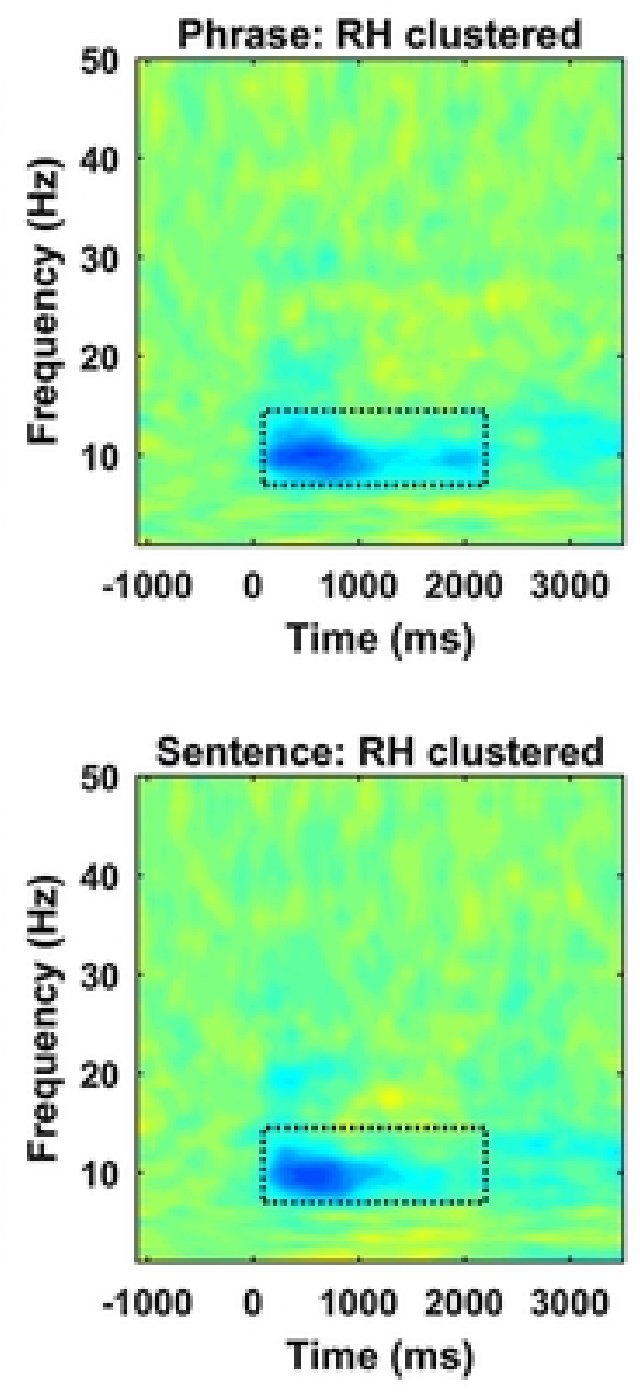

d

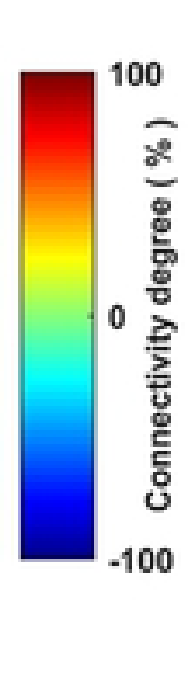

b
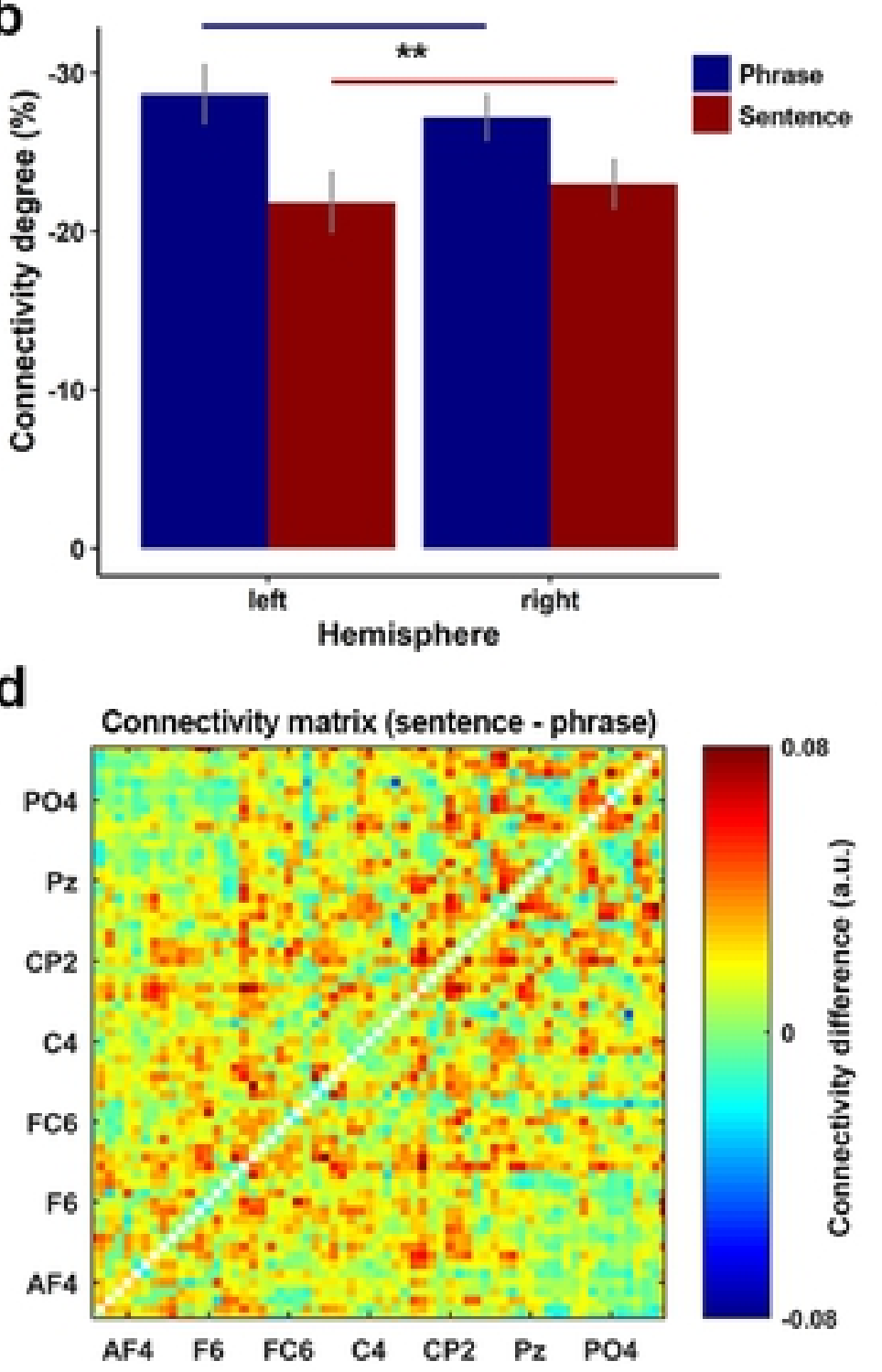

C

Selected sensors

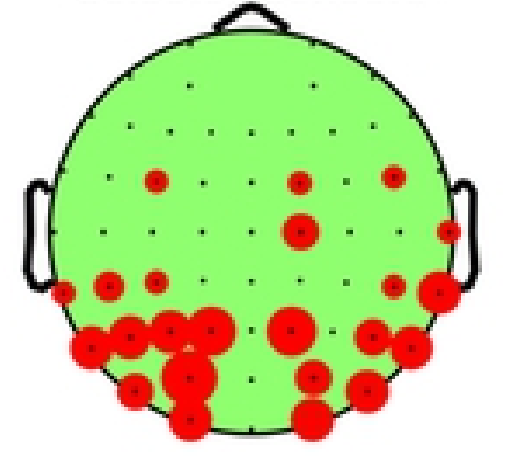

e

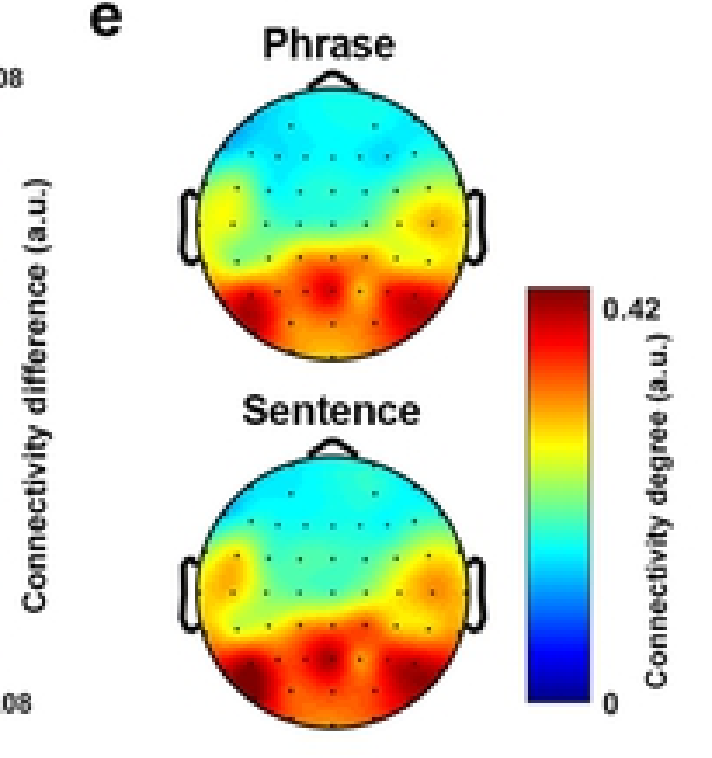

Figure 6 
a
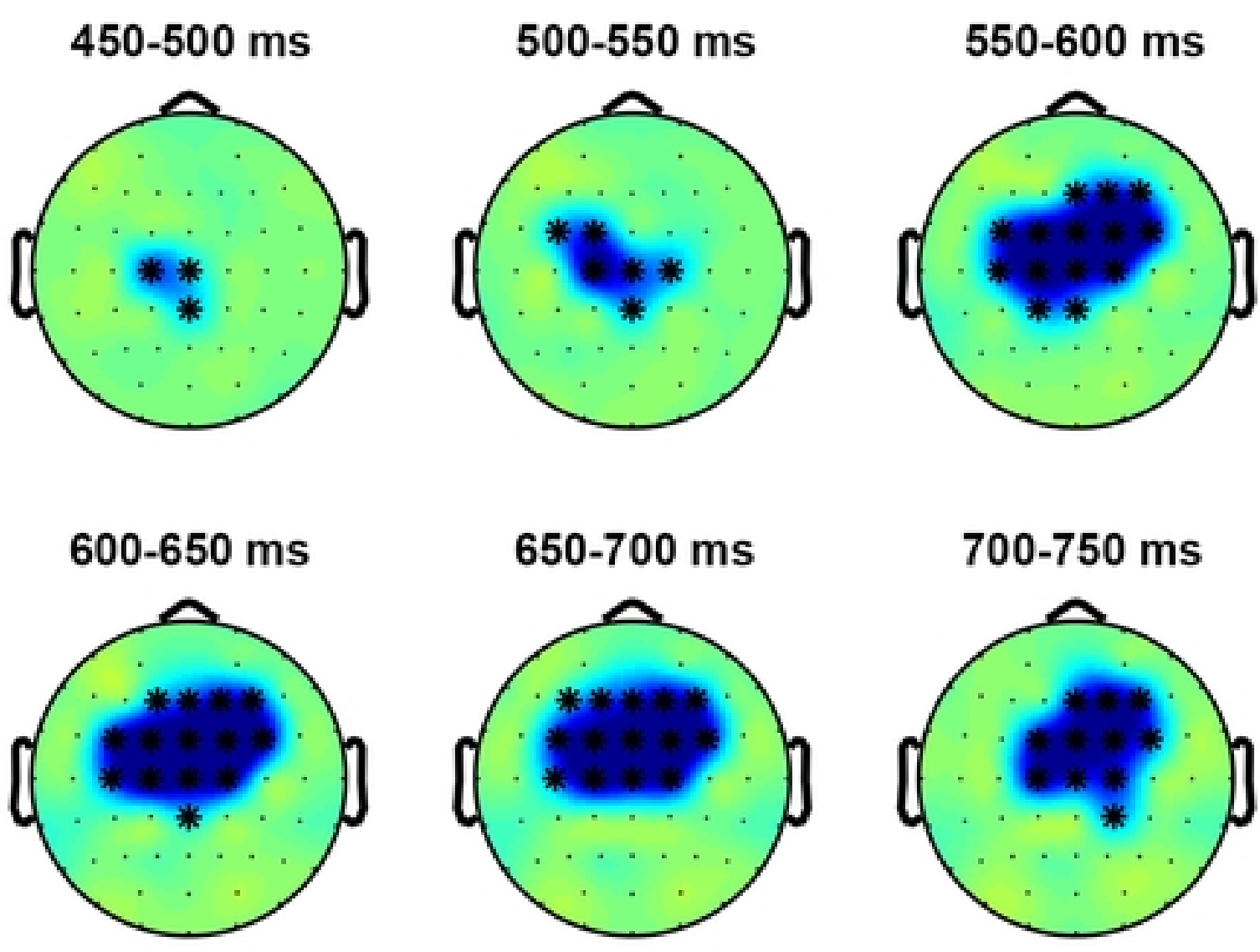

$750-800 \mathrm{~ms}$

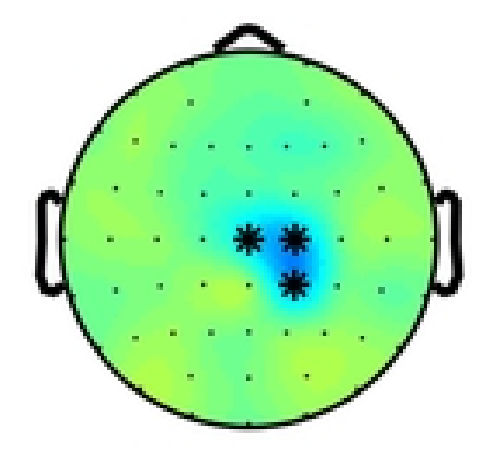

$800-850 \mathrm{~ms}$

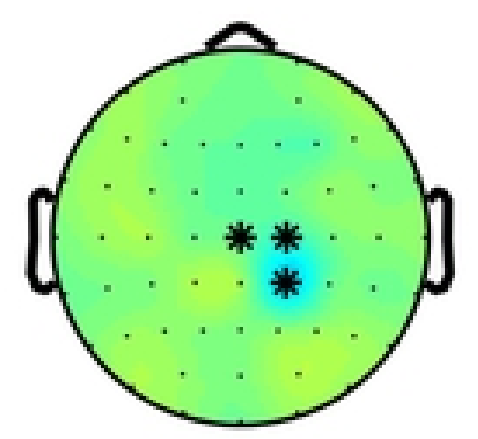

b
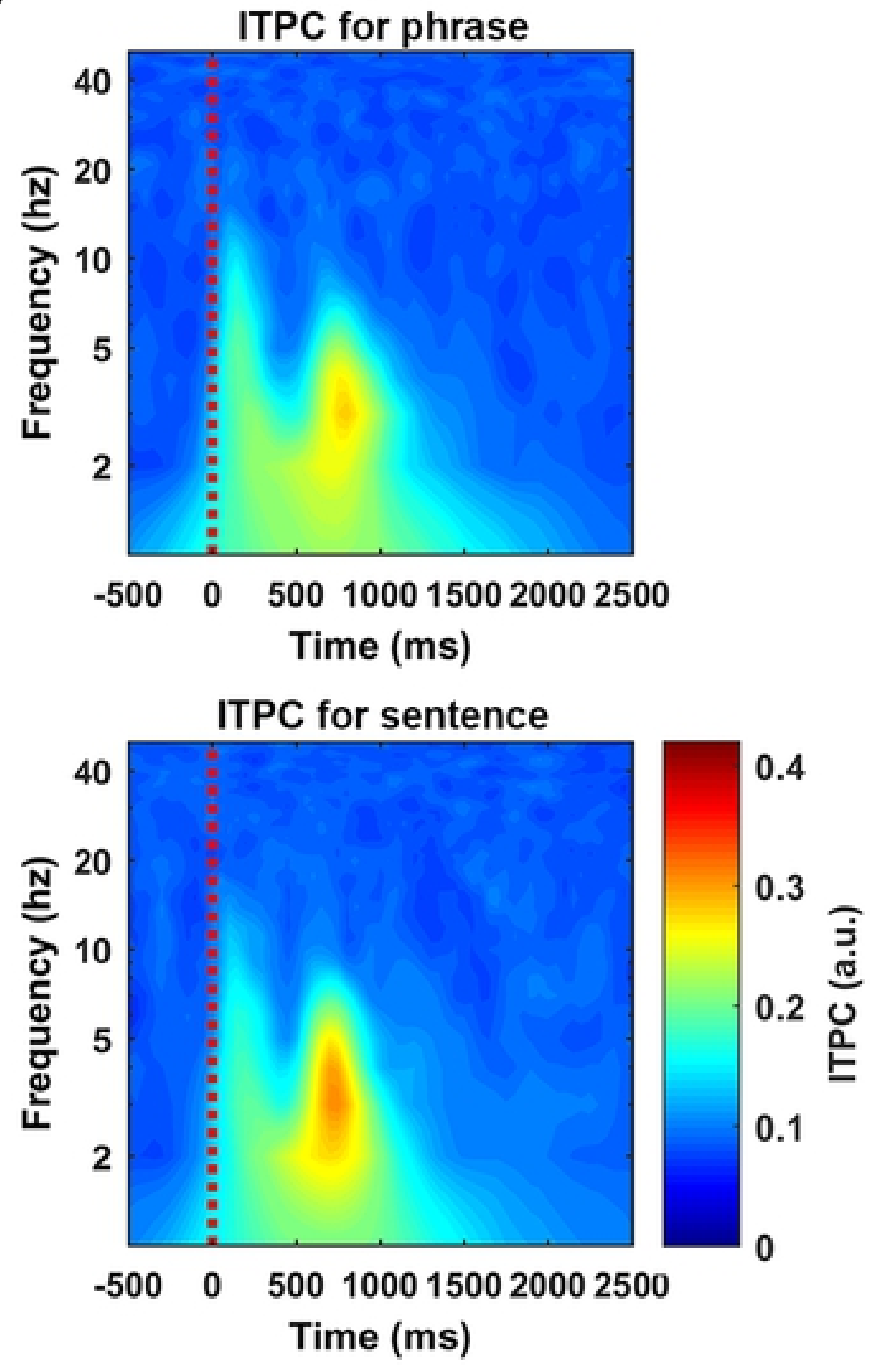

Figure 2 
a

$1800-1900 \mathrm{~ms} \quad 1900-2000 \mathrm{~ms} \quad 2000-2100 \mathrm{~ms} \quad 2100-2200 \mathrm{~ms}$
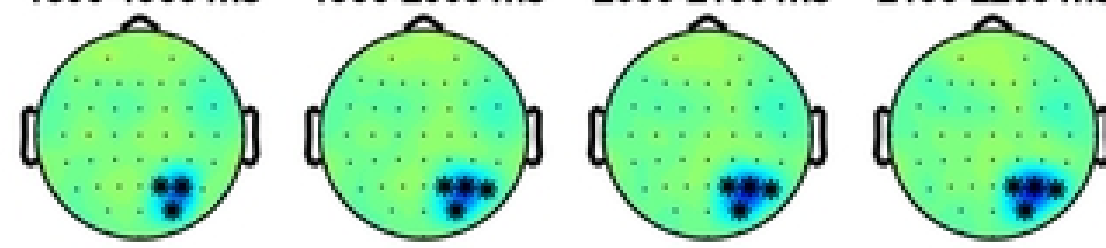

2200-2300 ms $2300-2400 \mathrm{~ms}$

2400-2500 ms

$2500-2600 \mathrm{~ms}$
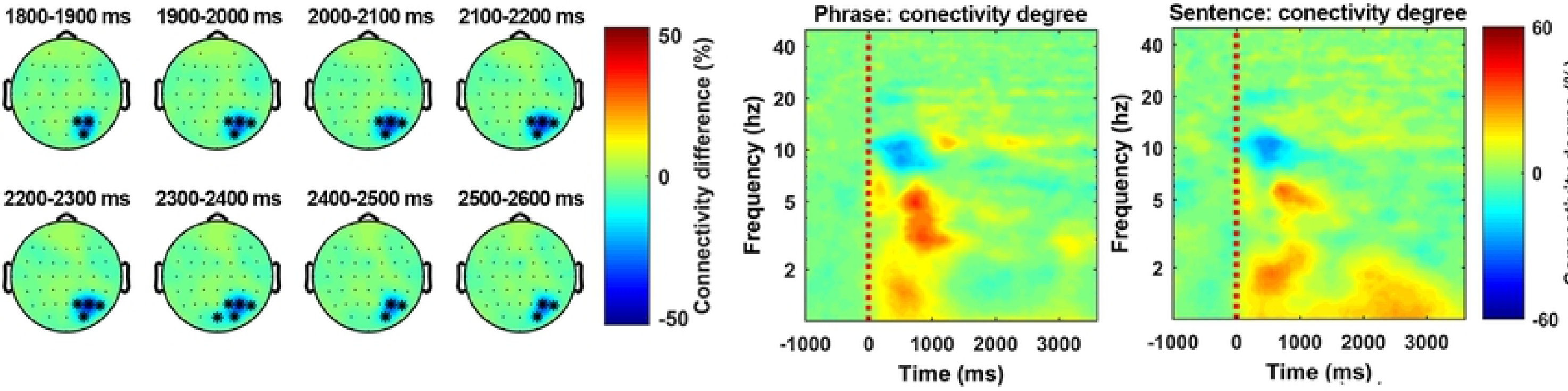

c

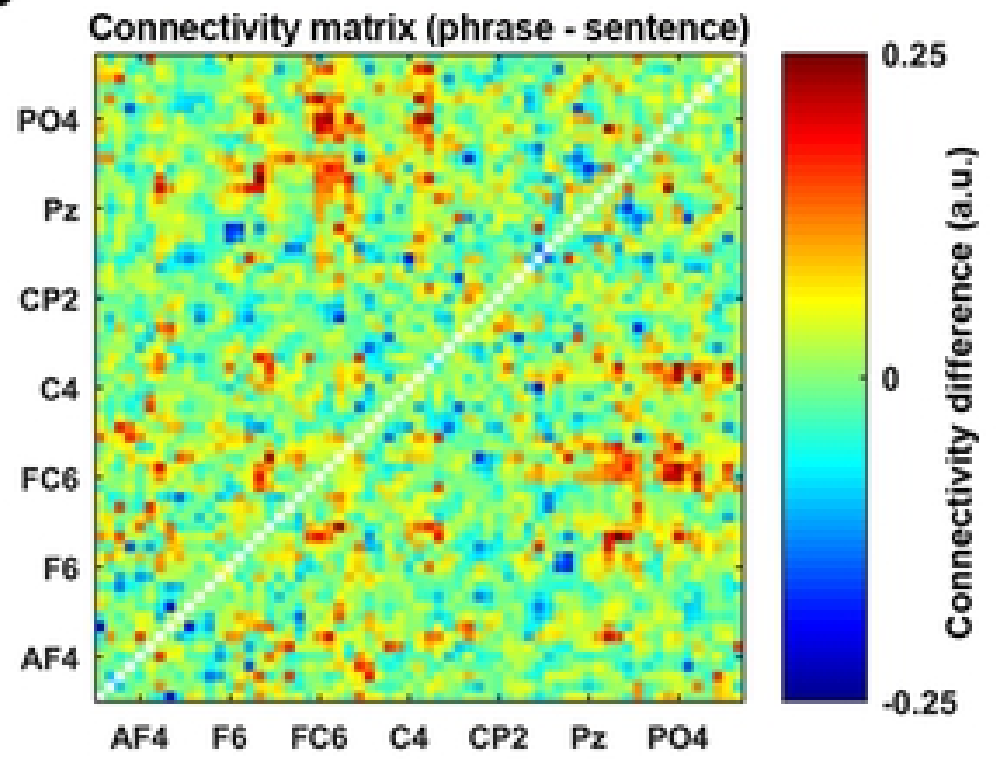

d

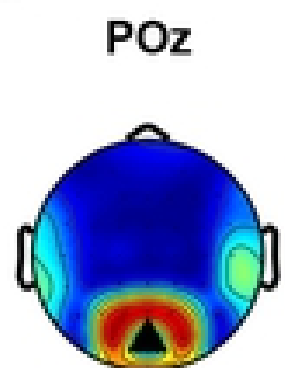

P2
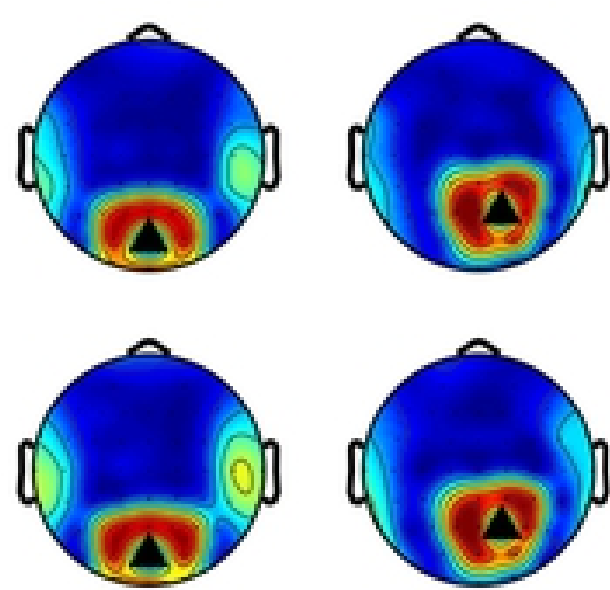

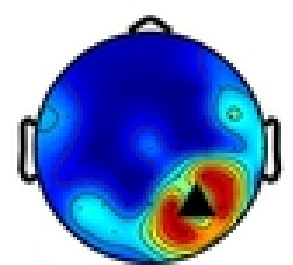

P6
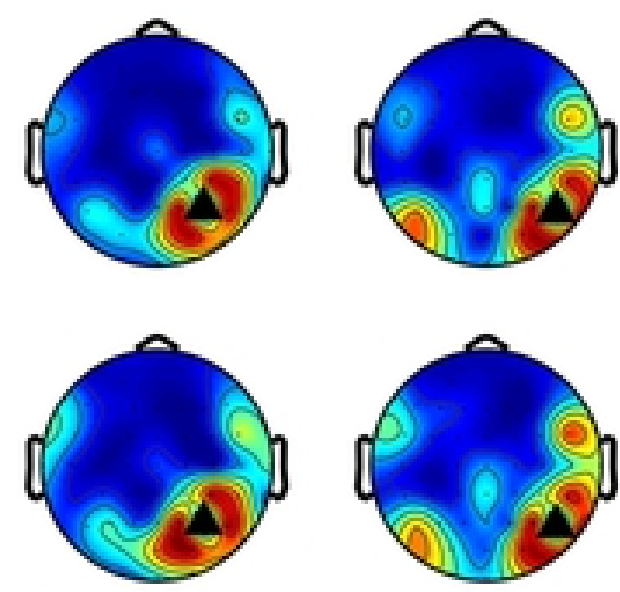

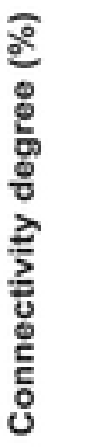

PO4
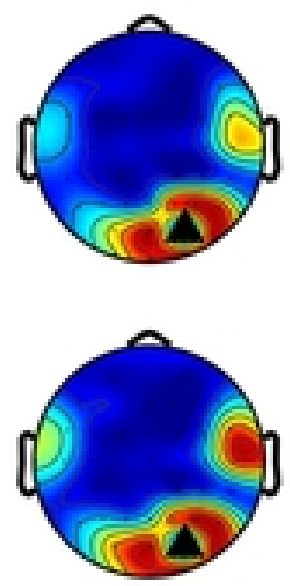

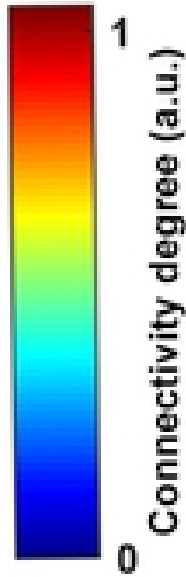

Figure 3 